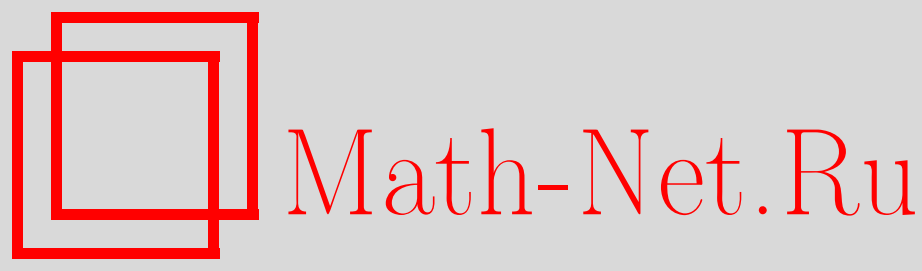

С. Е. Конштейн, А. Г. Смирнов, И. В. Тютин, Когомологии Хохшильда и деформации поточечного суперпроизведения, ТМФ, 2009, том 158, номер 3, 323-346

DOI: https://doi.org/10.4213/tmf6317

Использование Общероссийского математического портала Math-Net.Ru подразумевает, что вы прочитали и согласны с пользовательским соглашением http://www . mathnet.ru/rus/agreement

Параметры загрузки:

IP : 52.6 .47 .48

26 апреля 2023 г., 18:24:09

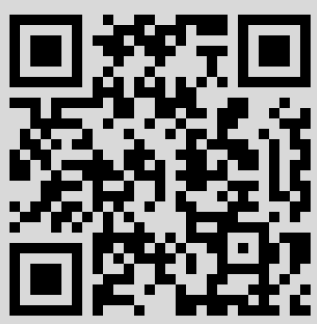




\title{
КОГОМОЛОГИИ ХОХШИЛЬДА И ДЕФОРМАЦИИ ПОТОЧЕЧНОГО СУПЕРПРОИЗВЕДЕНИЯ
}

\begin{abstract}
Рассмотрена ассоциативная супералгебра гладких грассманозначных функций с компактным носителем в $\mathbb{R}^{n}$. Найдены низшие когомологии Хохшильда, а также при некоторых дополнительных условиях деформации этой супералгебры.
\end{abstract}

Ключевые слова: деформационное квантование, квантовая механика, супералгебра, когомология Хохшильда.

\section{1. ВВЕДЕНИЕ}

Надежда построить квантовую механику на нетривиальных многообразиях связана с геометрическим, или деформационным, квантованием [1]-[4]. Функции на фазовом пространстве ассоциируются с операторами, а произведение и коммутатор операторов описываются ассоциативным *-произведением и *-коммутатором функций, которые являются деформациями обычного произведения и обычной скобки Пуассона.

Калибровочные теории на некоммутативных пространствах (так называемые некоммутативные калибровочные теории) формулируются в терминах *-произведения (см. [5], [6] и содержащиеся там ссылки). Структура и свойства *-произведения на обычных (четных) многообразиях изучены детально (см. [2]-[4]). В то же время *-произведение на супермногообразиях исследовано еще недостаточно.

Легко формально обобщить некоммутативное произведение, предложенное в работе [7], на суперслучай [8], однако при этом возникают проблемы с единственностью *-произведения (единственностью деформации поточечного произведения). В работе [9] найден общий вид ассоциативного *-произведения на грассмановой алгебре с конечным числом образующих и доказана его единственность с точностью до подходящего преобразования эквивалентности. Интересно решить аналогичную задачу для функций от четных и нечетных переменных.

\footnotetext{
* Физический институт им. П. Н. Лебедева РАН, Москва, Россия.

E-mail: konstein@lpi.ru,smirnov@lpi.ru, tyutin@lpi.ru

${ }^{\dagger}$ IF University of Sao Paulo, Sao Paulo, Brazil
} 
Каждое *-произведение порождает *-коммутатор, но обратное неверно (см. обсуждение в [10]). Например, как следует из результатов настоящей работы, найденная в работе [11] деформация суперскобки Пуассона, отличная от суперскобки Мойяла, не порождена *-произведением.

Задача о нахождении *-произведения связана с задачей о вычислении когомологий Хохшильда. В случае коцепей, задаваемых полидифференциальными операторами, когомологии Хохшильда алгебры гладких функций на гладком многообразии давно известны (см., например, [12]): p-я группа когомологий совпадает с пространством антисимметричных $p$-векторных полей. Кроме того, известно [13], что в случае гладких функций на $\mathbb{R}^{n}$ когомологии Хохшильда не изменяются при переходе от дифференциальных коцепей к непрерывным коцепям (см. также [14], где приведено прямое вычисление когомологий для непрерывного случая).

В настоящей работе исследуются когомологии и деформации ассоциативной супералгебры гладких функций с компактным носителем на $\mathbb{R}^{n}$, принимающих значения в алгебре Грассмана, причем мы ограничиваемся случаем, когда параметры деформаций являются четными элементами алгебры Грассмана. В разделах $3-5$ найдены низшие (до второй) когомологии Хохшильда данной супералгебры в предположении непрерывности коцепей по каждому аргументу. При этом мы всюду ограничиваемся случаем четных коцепей, поскольку только четные коциклы играют роль при построении *-произведений с четными параметрами деформации. В разделе 6 получен общий вид формальной деформации рассматриваемой супералгебры (теорема 3).

\section{2. ОСНОВНЫЕ ОБОЗНАЧЕНИЯ}

Пусть поле $\mathbb{K}$ - это либо $\mathbb{R}$, либо $\mathbb{C}$. Обозначим через $\mathcal{D}\left(\mathbb{R}^{n}\right)$ пространство гладких $\mathbb{K}$-значных функций с компактным носителем на $\mathbb{R}^{n}$. Это пространство снабжено своей стандартной топологией: по определению последовательность $\varphi_{k} \in \mathcal{D}\left(\mathbb{R}^{n}\right)$ сходится к $\varphi \in \mathcal{D}\left(\mathbb{R}^{n}\right)$, если носители всех $\varphi_{k}$ содержатся в некотором компакте и последовательность $\partial^{\lambda} \varphi_{k}$ сходится равномерно к $\partial^{\lambda} \varphi$ для любого мультииндекса $\lambda$. Положим

$$
\mathbf{D}_{n_{+}}^{n_{-}}=\mathcal{D}\left(\mathbb{R}^{n_{+}}\right) \otimes \mathbb{G}^{n_{-}}, \quad \mathbf{D}_{n_{+}}^{\prime n_{-}}=\mathcal{D}^{\prime}\left(\mathbb{R}^{n_{+}}\right) \otimes \mathbb{G}^{n_{-}},
$$

где $\mathbb{G}^{n_{-}}$- алгебра Грассмана с $n_{-}$образующими, а $\mathcal{D}^{\prime}\left(\mathbb{R}^{n_{+}}\right)$- пространство непрерывных линейных функционалов на $\mathcal{D}\left(\mathbb{R}^{n_{+}}\right)$. Образующие алгебры Грассмана и координаты пространства $\mathbb{R}^{n_{+}}$обозначаются через $\xi^{\alpha}, \alpha=1, \ldots, n_{-}$, и $x^{i}, i=1, \ldots, n_{+}$, соответственно. Мы будем также использовать коллективные переменные $z^{A}$, которые равны $x^{A}$ при $A=1, \ldots, n_{+}$и равны $\xi^{A-n_{+}}$при $A=n_{+}+1, \ldots, n_{+}+n_{-}$. Пространства $\mathbf{D}_{n_{+}}^{n_{-}}$имеют естественную градуировку, определенную градуировкой алгебры Грассмана. Четность элемента $f$ этого пространства обозначается через $\varepsilon(f)$. Положим также $\varepsilon_{A}=0$ для $A=1, \ldots, n_{+}$и $\varepsilon_{A}=1$ для $A=n_{+}+1, \ldots, n_{+}+n_{-}$.

Интеграл на пространстве $\mathbf{D}_{n_{+}}^{n_{-}}$определен соотношением

$$
\int d z f(z)=\int_{\mathbb{R}^{n^{+}}} d x \int d \xi f(z),
$$


где интеграл по алгебре Грассмана нормирован условием $\int d \xi \xi^{1} \ldots \xi^{n_{-}}=1$. Отождествим алгебру $\mathbb{G}^{n_{-}}$с ее дуальным пространством $\mathbb{G}^{\prime n_{-}}$, полагая $f(g)=\int d \xi f(\xi) g(\xi)$, $f, g \in \mathbb{G}^{n_{-}}$. Соответственно пространство $\mathbf{D}_{n_{+}}^{\prime n_{-}}$непрерывных линейных функционалов на $\mathbf{D}_{n_{+}}^{n_{-}}$отождествляется с пространством $\mathcal{D}^{\prime}\left(\mathbb{R}^{n_{+}}\right) \otimes \mathbb{G}^{n_{-}}$. Как правило, значение $m(f)$ функционала $m \in \mathbf{D}_{n_{+}}^{n_{-}}$на функциях $f \in \mathbf{D}_{n_{+}}^{n_{-}}$будет записываться в интегральной форме: $m(f)=\int d z m(z) f(z)$.

Назовем форму $\Phi$ суперантисимметричной, если

$$
\Phi(f, g)=-(-1)^{\varepsilon(f) \varepsilon(g)} \Phi(g, f) .
$$

Рассмотрим алгебру $\mathcal{A}=\sum_{\oplus} \mathcal{A}_{k}$, где $\mathcal{A}_{k}$ - векторное пространство четных $k$-линейных раздельно непрерывных форм $\Phi_{k} \in \mathcal{A}_{k}, k=0,1,2, \ldots$, принимающих значения в $\mathbf{D}_{n_{+}}^{n_{-}}, \varepsilon\left(\Phi_{k}\left(z \mid f_{1}, \ldots, f_{k}\right)\right)=\sum_{i=1}^{k} \varepsilon\left(f_{i}\right), \mathcal{A}_{0} \subset \mathbf{D}_{n_{+}}^{n_{-}}, \Phi_{0}(z)=\phi(z) \in \mathbf{D}_{n_{+}}^{n_{-}}$, $\varepsilon_{\Phi_{0}}=\varepsilon(\phi)=0$, с (некоммутативным) ассоциативным произведением

$$
\left(\Phi_{k} \diamond \Psi_{p}\right)\left(z \mid f_{1}, \ldots, f_{k+p}\right)=\Phi_{k}\left(z \mid f_{1}, \ldots, f_{k}\right) \Psi_{p}\left(z \mid f_{k+1}, \ldots, f_{k+p}\right) .
$$

Эта алгебра имеет естественную градуировку $g: g\left(\Phi_{k}\right)=k, g\left(\mathcal{A}_{k}\right)=k$ и дифференциал Хохшильда $d: \mathcal{A}_{k} \rightarrow \mathcal{A}_{k+1}, g(d)=1$, который действует по правилу

$$
\begin{aligned}
d_{k} \Phi_{k}\left(z \mid f_{1}, \ldots, f_{k+1}\right)= & f_{1}(z) \Phi_{k}\left(z \mid f_{2}, \ldots, f_{k+1}\right)+ \\
& +\sum_{i=1}^{k}(-1)^{i} \Phi_{k}\left(z \mid f_{1}, \ldots, f_{i-1}, f_{i}, f_{i+1}, f_{i+2}, \ldots, f_{k+1}\right)+ \\
& +(-1)^{k+1} \Phi_{k}\left(z \mid f_{1}, \ldots, f_{k}\right) f_{k+1}(z)
\end{aligned}
$$

Здесь $\left.d_{k} \stackrel{\text { def }}{=} d\right|_{\mathcal{A}_{k}}$. Дифференциал $d$ имеет следующие очевидные свойства:

$$
\begin{aligned}
d_{0} \Phi_{0}(z \mid f) & =f(z) \phi(z)-\phi(z) f(z)=0, \\
d_{k+p}\left(\Phi_{k} \Psi_{p}\right) & =\left(d_{k} \Phi_{k}\right) \Psi_{p}+(-1)^{g\left(\Phi_{k}\right)} \Phi_{k} d_{p} \Psi_{p}, \\
d_{k+1} d_{k} & =0, \quad k=0,1, \ldots
\end{aligned}
$$

Далее мы будем использовать следующие обозначения для многоиндексных объектов:

$$
\begin{gathered}
\left(\partial_{A}\right)^{k}=\partial_{A_{k}} \cdots \partial_{A_{1}}, \quad\left(p_{A}\right)^{k}=p_{A_{k}} \cdots p_{A_{1}} \\
\left(\overleftarrow{\partial}_{A}\right)^{k}=\overleftarrow{\partial}_{A_{1}} \cdots \overleftarrow{\partial}_{A_{k}}, \quad\left(\overleftarrow{p}_{A}\right)^{k}=p_{A_{1}} \cdots p_{A_{k}} \\
m^{(A)_{k}}=m^{A_{1} \cdots A_{k}}, \quad m^{\cdots A_{i} A_{i+1} \cdots}=(-1)^{\varepsilon_{A_{i}} \varepsilon_{A_{i+1}}} m^{\cdots A_{i+1} A_{i} \cdots}
\end{gathered}
$$

\section{3. КОГОМОЛОГИИ ХОХШИЛЬДА $H^{0}$}

Когомологическое уравнение

$$
d_{0} \Phi_{0}(z \mid f)=0
$$

удовлетворяется тождественно для произвольной формы $\Phi_{0}(z)$, так что мы имеем $H^{0}=\mathcal{A}_{0}$. 


\section{4. КОГОМОЛОГИИ ХОХШИЛЬДА $H^{1}$}

В этом случае когомологическое уравнение имеет вид

$$
f(z) \Phi_{1}(z \mid g)-\Phi_{1}(z \mid f g)+\Phi_{1}(z \mid f) g(z)=0 .
$$

Пусть $^{1)}$

$$
[z \cup \operatorname{supp}(g)] \cap \operatorname{supp}(f)=\varnothing \text {. }
$$

Из уравнения (4.1) имеем

$$
\widehat{\Phi}_{1}(z \mid f)=0,
$$

откуда следует ${ }^{2)}$

$$
\Phi_{1}(z \mid f)=\sum_{q=0}^{Q} m(z)^{(A)_{q}}\left(\partial_{A}\right)^{q} f(z),
$$

где $\widehat{\Phi}_{1}(z \mid f)$ обозначает ограничение линейной формы $\Phi_{1}(z \mid f)$ на область $(4.2)$ или, эквивалентно, на область $z \cap \operatorname{supp}(f)=\varnothing$. Здесь и ниже $Q$ локально постоянна.

Так же, как и в работах [15], [16], положим $f(z)=e^{z p}, g(z)=e^{z k}$ в некоторой окрестности точки $x$. Из уравнения (4.1) имеем

$$
F(z \mid k)-F(z \mid p+k)+F(z \mid p)=0
$$

где

$$
F(z \mid p)=\sum_{q=0}^{Q} m(z)^{(A)_{q}}\left(p_{A}\right)^{q},
$$

а уравнение (4.3) дает $F(z \mid p)=m(z)^{A} p_{A}$.

Таким образом, общее решение уравнения (4.1) имеет вид

$$
\Phi_{1}(z \mid f)=m(z)^{A} \partial_{A} f(z) .
$$

Очевидно, что различные $m(z)^{A}$ определяют независимые коциклы (4.4).

\section{5. КОГОМОЛОГИЯ ХОХШИЛЬДА $H^{2}$}

В этом разделе найдена вторая когомология Хохшильда для рассматриваемой супералгебры.

Решение когомологического уравнения

$$
f(z) \Phi_{2}(z \mid g, h)-\Phi_{2}(z \mid f g, h)+\Phi_{2}(z \mid f, g h)-\Phi_{2}(z \mid f, g) h(z)=0
$$

определяется ниже формулой (5.21).

Пусть $\mathcal{L}_{k} \subset \mathcal{A}_{k}$ обозначает пространство локальных форм. Форма $\Phi_{k} \in \mathcal{A}_{k}$ называется локальной, если из условия $z \bigcap \operatorname{supp}\left(f_{i}\right)=\varnothing$ при каком-либо $1 \leqslant i \leqslant k$ следует $\Phi_{k}\left(z \mid f_{1}, \ldots, f_{k}\right)=0$.

\footnotetext{
${ }^{1)}$ Пусть $z=(x, \xi)$. Здесь и далее мы используем обозначения $z \cup U \quad(z \cap U)$ вместо $V_{z} \cup U$ $\left(V_{z} \cap U\right)$, где $V_{z}$ - некоторая окрестность точки $x$.

${ }^{2)}$ Подробнее см. в работе [15].
} 


\section{1. Нелокальная часть коцикла. Пусть}

$$
[z \cup \operatorname{supp}(h)] \cap \operatorname{supp}(f)=[z \cup \operatorname{supp}(h)] \cap \operatorname{supp}(g)=\operatorname{supp}(f) \cap \operatorname{supp}(g)=\varnothing .
$$

Из уравнения (5.1) мы получаем

$$
\widehat{\Phi}_{2}(z \mid f, g)=0
$$

что влечет

$$
\begin{aligned}
\Phi_{2}(z \mid f, g)= & \sum_{q=0}^{Q}\left\{m_{1}^{(A)_{q}}(z \mid f)\left(\partial_{A}\right)^{q} g(z)+\right. \\
& \left.+\left[\left(\partial_{A}\right)^{q} f(z)\right] m_{2}^{(A)_{q}}(z \mid g)+m_{3}^{(A)_{q}}\left(z \mid f\left(\partial_{A}\right)^{q} g\right)\right\}
\end{aligned}
$$

где $\widehat{\Phi}_{2}(z \mid f, g)$ обозначает недиагональную часть формы $\Phi_{2}(z \mid f, g)$, т.е. ограничение формы $\Phi$ на область (5.2).

Заметим, что все формы $m_{a}^{(A)_{q}}$, в частности форма $m_{1}^{(A)_{0}}$, являются глобально определенными обобщенными функциями.

Рассмотрим область

$$
[z \cup \operatorname{supp}(f) \cup \operatorname{supp}(h)] \cap \operatorname{supp}(g)=\varnothing .
$$

Из уравнения (5.1) мы получаем следующее уравнение для ограничения формы $\Phi$ на область (5.4):

$$
f(z) \widehat{\Phi}_{2}(z \mid g, h)-\widehat{\Phi}_{2}(z \mid f, g) h(z)=0 .
$$

Подставляя представление (5.3) в уравнение (5.5), находим

$$
\sum_{q=0}^{Q}\left\{f(z) \widehat{m}_{1}^{(A)_{q}}(z \mid g)\left(\partial_{A}\right)^{q} h(z)-\left[\left(\partial_{A}\right)^{q} f(z)\right] \widehat{m}_{2}^{(A)_{q}}(z \mid g) h(z)\right\}=0,
$$

откуда следует

$$
\begin{gathered}
m_{1}^{(A)_{q}}(z \mid g) \in \mathcal{L}_{1}, \quad m_{2}^{(A)_{q}}(z \mid g) \in \mathcal{L}_{1}, \quad q \geqslant 1, \\
m_{2}^{(A)_{0}}(z \mid g)-m_{1}^{(A)_{0}}(z \mid g) \in \mathcal{L}_{1} .
\end{gathered}
$$

Таким образом, форма $\Phi_{2}(z \mid f, g)$ может быть представлена в виде

$$
\begin{aligned}
\Phi_{2}(z \mid f, g)= & {\left[\sum_{q=0}^{Q} m_{3}^{(A)_{q}}\left(z \mid f\left(\partial_{A}\right)^{q} g\right)+m_{1}^{(A)_{0}}(z \mid f g)\right]+} \\
& +\left[f(z) m_{1}^{(A)_{0}}(z \mid g)-m_{1}^{(A)_{0}}(z \mid f g)+\right. \\
& \left.+m_{1}^{(A)_{0}}(z \mid f) g(z)\right]+\Phi_{\text {loc }}(z \mid f, g), \quad \Phi_{\text {loc }}(z \mid f, g) \in \mathcal{L}_{2},
\end{aligned}
$$

или, после переопределения $m_{3}^{(A)_{0}}(z \mid f)$,

$$
\Phi_{2}(z \mid f, g)=\Phi_{2 \mid 3}(z \mid f, g)+d_{1} \Phi_{1 \mid 1}(z \mid f, g)+\Phi_{\text {loc }}(z \mid f, g),
$$


где

$$
\begin{aligned}
\Phi_{1 \mid 1}(z \mid f) & =m_{1}^{(A)_{0}}(z \mid f), \quad \varepsilon_{\Phi_{1 \mid 1}}=0, \\
\Phi_{2 \mid 3}(z \mid f, g) & =\sum_{q=0}^{Q} m_{3}^{(A)_{q}}\left(z \mid f\left(\partial_{A}\right)^{q} g\right) .
\end{aligned}
$$

Для того чтобы определить $\Phi_{2 \mid 3}$, рассмотрим область

$$
z \cap[\operatorname{supp}(f) \cup \operatorname{supp}(g) \cup \operatorname{supp}(h)]=\varnothing .
$$

Согласно представлению (5.6) из уравнения (5.1) получаем

$$
\widehat{\Phi}_{2 \mid 3}(z \mid f g, h)-\widehat{\Phi}_{2 \mid 3}(z \mid f, g h)=0 .
$$

Подставляя представление (5.8) в уравнение (5.10), находим

$$
\sum_{q=0}^{Q} \widehat{m}_{3}^{(A)_{q}}\left(z \mid\left\{f g\left(\partial_{A}\right)^{q} h-f\left(\partial_{A}\right)^{q}(g h)\right\}\right)=0 .
$$

Выбирая $g(z)=e^{-z p}, h(z)=e^{z p}$ на $\operatorname{supp}(f)$, получаем равенство

$$
\widehat{F}(z \mid f ; p)-\widehat{F}(z \mid f ; 0)=0, \quad F(z \mid f ; p)=\sum_{q=0}^{Q} \widehat{m}_{3}^{(A)_{q}}(z \mid f)\left(p_{A}\right)^{q}
$$

и затем

$$
\widehat{m}_{3}^{(A)_{q}}(z \mid f)=0 \Rightarrow m_{3}^{(A)_{q}}(z \mid f) \in \mathcal{L}_{1}, \quad q \geqslant 1 .
$$

Таким образом, форма $\Phi_{2}(z \mid f, g)$ может быть представлена в виде

$$
\begin{aligned}
\Phi_{2}(z \mid f, g) & =m(z \mid f g)+d_{1} \Phi_{1 \mid 1}(z \mid f, g)+\Phi_{\text {loc }}(z \mid f, g), \\
m(z \mid f) & =m_{3}^{(A)_{0}}(z \mid f) .
\end{aligned}
$$

Рассмотрим, наконец, область

$$
[z \cup \operatorname{supp}(h)] \cap[\operatorname{supp}(f) \cup \operatorname{supp}(g)]=\varnothing .
$$

Используя представление (5.11), из уравнения (5.1) находим

$$
m(z \mid f g h)+m(z \mid f g) h(z)=0, \quad m(z \mid f)=0 .
$$

Окончательно получаем

$$
\Phi_{2}(z \mid f, g)=\Phi_{2 \mid \text { loc }}(z \mid f, g)+d_{1} \Phi_{1 \mid 1}(z \mid f, g),
$$

где

$$
\Phi_{2 \mid \mathrm{loc}}(z \mid f, g)=\sum_{k, l=0}^{Q} f(z)\left(\overleftarrow{\partial}_{A}\right)^{k} m^{(A)_{k} \mid(B)_{l}}(z)\left(\partial_{B}\right)^{l} g(z), \quad \varepsilon_{m^{(A)_{k} \mid(B)_{l}}}=\varepsilon_{A_{1}}+\cdots+\varepsilon_{B_{l}}
$$

а все коэффициенты $m^{(A)_{k} \mid(B)_{l}}(z)$ и форма $\Phi_{1 \mid 1}(z \mid f)$ определены глобально.

Таким образом, всякий коцикл когомологичен локальному коциклу, так как нелокальные части коциклов являются точными формами. 
5.2. Локальные коциклы. Когомологическое уравнение (5.1) принимает следующий вид для локальных форм:

$$
\begin{aligned}
\sum_{k, l=0}^{Q} & \left(f(z)\left[g(z)\left(\overleftarrow{\partial}_{A}\right)^{k}\right] m^{(A)_{k} \mid(B)_{l}}(z)\left(\partial_{B}\right)^{l} h(z)-\right. \\
& -[f(z) g(x)]\left(\overleftarrow{\partial}_{A}\right)^{k} m^{(A)_{k} \mid(B)_{l}}(z)\left(\partial_{B}\right)^{l} h(z)+ \\
& +f(z)\left(\overleftarrow{\partial}_{A}\right)^{k} m^{(A)_{k} \mid(B)_{l}}(z)\left(\partial_{B}\right)^{l}[g(z) h(z)]- \\
& \left.-f(z)\left(\overleftarrow{\partial}_{A}\right)^{k} m^{(A)_{k} \mid(B)_{l}}(z)\left[\left(\partial_{B}\right)^{l} g(z)\right] h(z)\right)=0
\end{aligned}
$$

Пусть $f(z)=e^{p z}, g(z)=e^{q z}, h(z)=e^{r z}$ в некоторой окрестности точки $x$. Тогда когомологическое уравнение преобразуется к виду

$$
\begin{gathered}
F(z \mid q, \tilde{r})-F(z \mid p+q, \tilde{r})+F(z \mid p, \tilde{q}+\tilde{r})-F(z \mid p, \tilde{q})=0 \\
F(z \mid p, q)=\sum_{k, l=0}^{Q}\left(p_{A}\right)^{k} m^{(A)_{k} \mid(B)_{l}}(z)\left(q_{B}\right)^{l}, \quad \tilde{p}_{A}=(-1)^{\varepsilon_{A}} p_{A} .
\end{gathered}
$$

Применяя оператор $\partial /\left.\partial p_{A}\right|_{p=0}$ к уравнению (5.15), получаем

$$
\begin{aligned}
\frac{\partial}{\partial q_{A}} F(z \mid q, \tilde{r}) & =\Psi^{A}(z \mid q+r)-\Psi^{A}(z \mid q), \\
\Psi^{A}(z \mid q) & =\left.\frac{\partial}{\partial p_{A}} F(z \mid p, \tilde{q})\right|_{p=0} .
\end{aligned}
$$

Из уравнения (5.16) следует

$$
\begin{gathered}
\frac{\partial}{\partial q_{A}} \Psi^{B}(z \mid q+r)-(-1)^{\varepsilon_{A} \varepsilon_{B}} \frac{\partial}{\partial q_{B}} \Psi^{A}(z \mid q+r)= \\
=\frac{\partial}{\partial q_{A}} \Psi^{B}(z \mid q)-(-1)^{\varepsilon_{A} \varepsilon_{B}} \frac{\partial}{\partial q_{B}} \Psi^{A}(z \mid q)
\end{gathered}
$$

и затем

$$
\begin{gathered}
\frac{\partial}{\partial r_{A}} \Psi^{B}(z \mid r)-(-1)^{\varepsilon_{A} \varepsilon_{B}} \frac{\partial}{\partial r_{B}} \Psi^{A}(z \mid r)=-(-1)^{\varepsilon_{A} \varepsilon_{B}} \omega^{A B}(z), \\
\omega^{A B}(z)=\frac{\partial}{\partial q_{B}} \Psi^{A}(z \mid q)-\left.(-1)^{\varepsilon_{A} \varepsilon_{B}} \frac{\partial}{\partial q_{A}} \Psi^{B}(z \mid q)\right|_{q=0}=-(-1)^{\varepsilon_{A} \varepsilon_{B}} \omega^{B A}(z) .
\end{gathered}
$$

Общее решение уравнения (5.18) имеет вид

$$
\Psi^{A}(z \mid r)=\frac{1}{2} \omega^{A B}(z) r_{B}+\frac{\partial}{\partial r_{A}} \phi(z \mid r) .
$$

Функция (5.19) также удовлетворяет уравнению (5.17). Подставляя выражение (5.19) в уравнение $(5.16)$, получаем

$$
\frac{\partial}{\partial q_{A}}\left(F(z \mid q, \tilde{r})-\frac{1}{2} q_{A} \omega^{A B}(z) r_{B}-\phi(z \mid q+r)+\phi(z \mid q)\right)=0
$$


и, как следствие,

$$
F(z \mid q, r)=\frac{1}{2} q_{A} \omega^{A B}(z) \tilde{r}_{B}+\phi(z \mid q+\tilde{r})-\phi(z \mid q)+\varphi(z \mid \tilde{r}) .
$$

Подставляя теперь (5.20) в уравнение (5.15), находим, что функция (5.20) удовлетворяет уравнению (5.15), если функция $\varphi(z \mid r)$ имеет вид $\varphi(z \mid r)=-\phi(z \mid r)-\varphi_{1}(z)$. Таким образом, получаем

$$
F(z \mid p, q)=\frac{1}{2} p_{A} \omega^{A B}(z) \tilde{q}_{B}+\phi(z \mid p+\tilde{q})-\phi(z \mid p)-\phi(z \mid \tilde{q})
$$

где было сделано переопределение $\phi(z \mid p) \rightarrow \phi(z \mid p)+\varphi_{1}(z)$, или

$$
\begin{aligned}
\Phi_{2 \mid \mathrm{loc}}(z \mid f, g) & =\frac{1}{2} f(z) \overleftarrow{\partial}_{A} \omega^{A B}(z) \partial_{B} g(z)+d_{1} \Phi_{1 \mid 2}(z \mid f, g) \\
\Phi_{1 \mid 2}(z \mid f) & =-f(z) \sum_{k=0}^{K}\left(\overleftarrow{\partial}_{A}\right)^{k} \phi^{(A)_{k}}(z)
\end{aligned}
$$

где $\phi^{(A)_{k}}(z)$ - коэффициенты полинома $\phi(z \mid p)=\sum_{k=0}^{K}\left(p_{A}\right)^{k} \phi^{(A)_{k}}(z)$.

Окончательно, с учетом (5.13) мы находим, что общее решение когомологического уравнения (5.1) имеет вид

$$
\Phi_{2}(z \mid f, g)=m_{\omega}(z \mid f, g)+d_{1} \Phi_{1}(z \mid f, g),
$$

где

$$
\begin{gathered}
m_{\omega}(z \mid f, g)=\frac{1}{2} f(z) \overleftarrow{\partial}_{A} \omega^{A B}(z) \partial_{B} g(z)=-(-1)^{\varepsilon(f) \varepsilon(g)} m_{\omega}(z \mid g, f) \\
\varepsilon\left(\omega^{A B}\right)=\varepsilon_{A}+\varepsilon_{B} .
\end{gathered}
$$

Когомологии с различными $\omega^{A B}(z)$ независимы. Действительно, рассмотрим уравнение

$$
\begin{aligned}
f(z) \overleftarrow{\partial}_{A} \omega^{A B}(z) \partial_{B} g(z) & =d_{1} \Psi_{1}(z \mid f, g)= \\
& =f(z) \Psi_{1}(z \mid g)-\Psi_{1}(z \mid f g)+\Psi_{1}(z \mid f) g(z)
\end{aligned}
$$

Пусть

$$
[z \cup \operatorname{supp}(g)] \cap \operatorname{supp}(f)=\varnothing .
$$

Из уравнения (5.23) находим

$$
\widehat{\Psi}_{1}(z \mid f)=0
$$

т.е. форма $\Psi_{1}(z \mid f)$ является локальной:

$$
\Psi_{1}(z \mid f)=f(z) \sum_{k=0}^{K}\left(\overleftarrow{\partial}_{A}\right)^{k} \psi^{(A)_{k}}(z) \equiv f(z) \psi\left(\overleftarrow{\partial}_{A} \mid z\right)
$$

Пусть $f(z)=e^{p z}, g(z)=e^{q z}$. Из уравнения (5.23) следует

$$
p_{A} \omega^{A B}(z)(-1)^{\varepsilon_{B}} q_{B}=\psi(q \mid z)-\psi(p+q \mid z)+\psi(p \mid z) .
$$


Правая часть этого уравнения симметрична относительно перестановки $p \leftrightarrow q$, так что мы имеем

$$
p_{A} \omega^{A B}(z)(-1)^{\varepsilon_{B}} q_{B}=q_{B} \omega^{B A}(z)(-1)^{\varepsilon_{A}} p_{A}=-p_{A} \omega^{A B}(z)(-1)^{\varepsilon_{B}} q_{B},
$$

откуда следует $\omega^{A B}(z)=0$. Тем самым, уравнение (5.23) имеет решения только при $\omega^{A B}(z)=0$.

5.3. Компактность и гладкость. Заметим, что слагаемые, стоящие в правой части выражения (5.21), обладают следующими свойствами суперсимметрии:

$$
\begin{aligned}
m_{\omega}(z \mid f, g) & =-(-1)^{\varepsilon(f) \varepsilon(g)} m_{\omega}(z \mid g, f), \\
\Phi_{d 1}(z \mid f, g) & \equiv d_{1} \Phi_{1}(z \mid f, g)=(-1)^{\varepsilon(f) \varepsilon(g)} \Phi_{d 1}(z \mid g, f) .
\end{aligned}
$$

Таким образом, имеют место равенства

$$
\begin{aligned}
& \Phi_{2}(z \mid f, g)-(-1)^{\varepsilon(f) \varepsilon(g)} \Phi_{2}(z \mid g, f)=2 m_{\omega}(z \mid f, g), \\
& \Phi_{2}(z \mid f, g)+(-1)^{\varepsilon(f) \varepsilon(g)} \Phi_{2}(z \mid g, f)=2 \Phi_{d 1}(z \mid f, g),
\end{aligned}
$$

откуда следует, что каждая из форм $m_{\omega}(z \mid f, g)$ и $\Phi_{d 1}(z \mid f, g)$ должна иметь компактный носитель и быть гладкой по $z$ при любых $f$ и $g$ из $\mathbf{D}_{n_{+}}^{n_{-}}$.

Из гладкости формы $m_{\omega}(z \mid f, g)$ вытекает гладкость суперсимплектической метрики $\omega^{A B}(z)$ (достаточно рассмотреть, например, $f(z)=z^{A}, g(z)=z^{B}$ в некоторой окрестности точки $x)$.

Рассмотрим условия компактности и гладкости формы

$$
\Phi_{d 1}(z \mid f, g)=f(z) \Phi_{1}(z \mid g)+\Phi_{1}(z \mid f) g(z)-\Phi_{1}(z \mid f g) \text {. }
$$

Поскольку первые два слагаемых в правой части выражения (5.24) имеют компактные носители, третье слагаемое (т.е. форма $\Phi_{1}(z \mid f)$ ) также должно иметь компактный носитель. Кроме того, из леммы 8, доказанной ниже в приложении А, следует, что форма $\Phi_{1}(z \mid f)$ является гладкой с точностью до слагаемого с нулевым дифференциалом для любой функции $f \in \mathbf{D}_{n_{+}}^{n_{-}}$. Таким образом, $\Phi_{2}(z \mid f, g)$ можно представить в виде (5.21), где форма $\Phi_{1}(z \mid f)$ при любых $f \in \mathbf{D}_{n_{+}}^{n_{-}}$имеет компактный носитель и является гладкой, а суперсимплектическая метрика $\omega^{A B}(z)$ гладкая.

ПредПоложЕниЕ. Далее мъ везде будем предполагать, ито форма $\omega^{A B}(z)$ невърожденна при всех $z$.

\section{6. ДЕФОРМАЦИИ ПОТОЧЕЧНОГО СУПЕРПРОИЗВЕДЕНИЯ}

Рассмотрим четную $\left(\varepsilon_{\Pi}=0\right)$ деформацию поточечного произведения $(f * g)(z) \equiv$ $\Pi(z \mid f, g ; \hbar)=f(z) g(z)+O(\hbar)$ (звездочное произведение), рассматриваемого как формальный ряд по $\hbar$ и обладающего следующими свойствами:

1) звездочное произведение ассоциативно:

$$
f *(g * h)=(f * g) * h,
$$


или

$$
\Pi(z \mid f, \Pi(\mid g, h ; \hbar) ; \hbar)=\Pi(z \mid \Pi(\mid f, g ; \hbar), h ; \hbar)
$$

2) кроме того,

$$
\Pi(z \mid f, g ; \hbar) \in \mathbf{D}_{n_{+}}^{n_{-}} \quad \text { для всех } f, g, \in \mathbf{D}_{n_{+}}^{n_{-}} .
$$

В нулевом порядке по $\hbar$ уравнение ассоциативности очевидно удовлетворяется.

Преобразование $T: \quad f(z) \rightarrow T f=T(z \mid f)$ вида $T=I+\hbar T_{1}+\hbar^{2} T_{2}+\cdots$ будем называть преобразованием подобия, а формы П $(z \mid f, g ; \hbar)$ и $\Pi_{T}(z \mid f, g ; \hbar)=$ $T^{-1} \Pi(z \mid T f, T g ; \hbar)$ - подобными формами. Очевидно, что формы П и П менно удовлетворяют или одновременно не удовлетворяют уравнению ассоциативности.

Справедлива следующая

ЛЕмма 1. Пусть данъ 2-форма П и преобразование подобия $T=1+\hbar^{k} T_{k}+$ $O\left(\hbar^{k+1}\right)$. Тогда

$$
\Pi_{T}=\Pi+\hbar^{k} d_{1} T_{(k)}+O\left(\hbar^{k+1}\right) .
$$

6.1. Первый порядок. Для формы

$$
\Pi(z \mid f, g ; \hbar)=f(z) g(z)+\hbar \Pi_{(1)}(z \mid f, g)+O\left(\hbar^{2}\right), \quad \Pi_{(1)}(z \mid f, g) \in D,
$$

уравнение ассоциативности (6.1) дает в первом порядке по $\hbar$

$$
\begin{aligned}
d_{2} \Pi_{(1)}(z \mid f, g, h) & =0 \Rightarrow \\
\Rightarrow \Pi_{(1)}(z \mid f, g) & =m_{\omega_{1}}(z \mid f, g)+d_{1} M_{(1)}(z \mid f, g), \quad M_{(1)}(z \mid f) \in D .
\end{aligned}
$$

С помощью преобразования подобия $T=I-\hbar M_{(1)}(z \mid f)+O\left(\hbar^{2}\right)$ (индекс $T$ мы далее будем опускать), форма П может быть представлена в виде

$$
\Pi(z \mid f, g ; \hbar)=f(z) g(z)+\hbar m_{\omega_{1}}(z \mid f, g)+\hbar^{2} \Pi_{(2)}(z \mid f, g)+O\left(\hbar^{3}\right), \quad \Pi_{(2)}(z \mid f, g) \in D
$$

6.2. Вспомогательные леммы. Введем ассоциатор $\operatorname{Asc}\left(\Pi_{1}, \Pi_{2}\right)(z \mid f, g, h)$ двух 2-форм $\Pi_{1}$ и $\Pi_{2}$ :

$$
\operatorname{Asc}\left(\Pi_{1}, \Pi_{2}\right)(z \mid f, g, h)=\Pi_{1}\left(z \mid f, \Pi_{2}(\mid g, h ; \hbar) ; \hbar\right)-\Pi_{1}\left(z \mid \Pi_{2}(\mid f, g ; \hbar), h ; \hbar\right)+(1 \leftrightarrow 2) .
$$

В терминах ассоциаторов уравнение ассоциативности (6.1) имеет вид

$$
\frac{1}{2} \operatorname{Asc}(\Pi, \Pi)=0
$$

Утверждения следующих лемм проверяются непосредственными вычислениями.

Лемма 2. Пусть $\Pi=f g+\hbar m_{\omega_{1}}+\hbar^{2} \Pi_{(2)}+O\left(\hbar^{3}\right)$. Тогда

$$
\frac{1}{2} \operatorname{Asc}(\Pi, \Pi)=\hbar^{2} d_{2} \Pi_{(2)}+\frac{1}{2} \hbar^{2} \operatorname{Asc}\left(m_{\omega_{1}}, m_{\omega_{1}}\right)+O\left(\hbar^{3}\right) .
$$


Лемма 3. Пусть $\Pi=W+\hbar^{n} \Pi_{(n)}+\hbar^{n+1} \Pi_{(n+1)}+O\left(\hbar^{n+2}\right)$, где $W=f g+\hbar m_{\omega_{1}}+$ $O\left(\hbar^{2}\right)$, удовлетворяет уравнению ассочиативности (6.1). Тогда

$$
\frac{1}{2} \operatorname{Asc}(\Pi, \Pi)=\hbar^{n} d_{2} \Pi_{(n)}+\hbar^{n+1} d_{2} \Pi_{(n+1)}+\hbar^{n+1} \operatorname{Asc}\left(m_{\omega_{1}}, \Pi_{(n)}\right)+O\left(\hbar^{n+2}\right) .
$$

ЛЕмма 4. Для произволъной суперантисимметричной 2-формы П выполняется тождество

$$
\begin{aligned}
& (-1)^{\varepsilon(f) \varepsilon(h)} d_{2} \Pi(z \mid f, g, h)-(-1)^{\varepsilon(f) \varepsilon(h)+\varepsilon(f) \varepsilon(g)} d_{2} \Pi(z \mid g, f, h)+ \\
& \quad+\operatorname{cycle}(f, g, h) \equiv 0
\end{aligned}
$$

6.3. Второй порядок. Согласно лемме 2 во втором порядке по $\hbar$ уравнение ассоциативности (6.1) принимает вид

$$
d_{2} \Pi_{(2)}+\frac{1}{2} \operatorname{Asc}\left(m_{\omega_{1}}, m_{\omega_{1}}\right)=0 .
$$

Используя тождество (6.3), получаем (учитывая, что форма $m_{\omega_{1}}(z \mid f, g)$ суперантисимметрична)

$$
(-1)^{\varepsilon(f) \varepsilon(h)} m_{\omega_{1}}\left(z \mid f, m_{\omega_{1}}(\mid g, h)\right)+\operatorname{cycle}(f, g, h)=0 .
$$

В левой части этого равенства члены со вторыми производными одной из функций $f, g$ или $h$ тождественно сокращаются, а приравнивание нулю коэффициента перед $\partial f \partial g \partial h$ дает

$$
(-1)^{\varepsilon_{A} \varepsilon_{C}} \omega_{1}^{A D}(z) \partial_{D} \omega_{1}^{B C}+\operatorname{cycle}(f, g, h)=0 .
$$

Таким образом, $\omega_{1}^{A B}(z)$ суперантисимметрична и удовлетворяет тождеству Якоби, т.е. является метрикой суперскобки Пуассона.

Отметим, что матрица $\omega_{1 A B}(z)$, такая что $\omega_{1 A B} \omega_{1}^{B C}(z)=\delta_{A}^{C}$, удовлетворяет тождеству

$$
(-1)^{\left(1+\varepsilon_{A}\right) \varepsilon_{C}} \partial_{A}^{z} \omega_{1 B C}(z)+\operatorname{cycle}(A, B, C)=0 .
$$

В дальнейшем для каждой удовлетворяющей тождеству Якоби суперантисимметричной метрики

$$
\omega^{A B}(z)=\omega_{1}^{A B}(z)+\hbar \omega_{2}^{A B}(z)+\cdots,
$$

где матрица $\omega_{1}^{A B}(z)$ невырожденна при всех $z$, будет построено такое звездочное произведение $\Pi_{F \omega}$, что

$$
\Pi_{F \omega^{(1)}}-\Pi_{F \omega^{(2)}}=\hbar^{k} m_{\widetilde{\omega}_{k}}+O\left(\hbar^{k+1}\right), \quad \widetilde{\omega}=\omega^{(1)}-\omega^{(2)},
$$

для любых таких метрик $\omega^{(1)}$ и $\omega^{(2)}$, что $\omega^{(1)}=\omega^{(2)}\left(\bmod \hbar^{k}\right)$. Будет показано, что произвольная деформация $\Pi(z \mid f, g ; \hbar)=f g+\hbar m_{\omega_{1}}(z \mid f, g)+\cdots$ поточечного произведения, где матрица $\omega_{1}^{A B}(z)$ обратима при всех $z$, эквивалентна с точностью до преобразования подобия звездочному произведению $\Pi_{F \omega}(z \mid f, g ; \hbar)$, где $\omega-$ суперантисимметричная метрика вида (6.5), удовлетворяющая тождеству Якоби. Для построения $\Pi_{F \omega}$ нам потребуется адаптировать известную конструкцию Федосова [3] на суперслучай. 
6.4. Суперконструкция Федосова. Введем формальную вейлевскую супералгебру $W$ :

$$
\begin{gathered}
a \in W \Rightarrow a=a(z \mid y, \eta ; \hbar)=\sum_{p, q, k \geqslant 0} \hbar^{k} a_{k\left|(A)_{p}\right|(B)_{q}}(z)\left(y^{A}\right)^{p}\left(\eta^{B}\right)^{q}, \\
\varepsilon\left(z^{A}\right)=\varepsilon\left(y^{A}\right)=\varepsilon_{A}, \quad \varepsilon\left(\eta^{A}\right)=\varepsilon_{A}+1, \\
\operatorname{deg}\left(z^{A}\right)=\operatorname{deg}\left(\eta^{A}\right)=0, \quad \operatorname{deg}\left(y^{A}\right)=1, \quad \operatorname{deg}(\hbar)=2,
\end{gathered}
$$

с ассоциативным произведением

$$
\begin{gathered}
a \circ b(z \mid y, \eta ; \hbar)=a(z \mid y, \eta ; \hbar) e^{(i \hbar / 2) \widehat{\omega}} b(z \mid y, \eta ; \hbar)= \\
=(-1)^{\varepsilon(a) \varepsilon(b)} b(z \mid y, \eta ; \hbar) e^{(-i \hbar / 2) \widehat{\omega}} a(z \mid y, \eta ; \hbar) \\
\widehat{\omega}=\overleftarrow{\partial}_{A}^{y} \omega^{A B}(z) \partial_{B}^{y}, \quad \omega^{B A}(z)=-(-1)^{\varepsilon_{A} \varepsilon_{B}} \omega^{A B}(z), \quad \varepsilon\left(\omega^{A B}\right)=\varepsilon_{A}+\varepsilon_{B}, \\
(-1)^{\varepsilon_{A} \varepsilon_{C}} \omega^{A D}(z) \partial_{D}^{z} \omega^{D C}(z)+\operatorname{cycle}(A, B, C)=0 .
\end{gathered}
$$

Суперкоммутатором $[a, b]$ назовем выражение

$$
[a, b]=a \circ b-(-1)^{\varepsilon(a) \varepsilon(b)} b \circ a=2 i a \sin \left(\frac{\hbar}{2} \widehat{\omega}\right) b, \quad a, b \in W,
$$

обладающее следующими свойствами:

1) суперантисимметрия

$$
[b, a]=-(-1)^{\varepsilon(a) \varepsilon(b)}[b, a]
$$

2) правило Лейбница

$$
[a, b \circ c]=[a, b] \circ c+(-1)^{\varepsilon(a) \varepsilon(b)} b \circ[a, c], \quad a, b, c \in W
$$

3) тождество Якоби

$$
(-1)^{\varepsilon(a) \varepsilon(c)}[a,[b, c]]+\operatorname{cycle}(a, b, c)=0, \quad a, b, c \in W .
$$

Для функции $a \in W$ введем обозначения

$$
a_{0}=a_{0}(z \mid \eta ; \hbar)=\left.a(z \mid y, \eta ; \hbar)\right|_{y=0}, \quad a_{00}=a_{00}(z \mid \hbar)=\left.a(z \mid y, \eta ; \hbar)\right|_{y=\eta=0} .
$$

Подмножество функций $W_{Z}$ супералгебры $W$, коммутирующих со всеми функциями из супералгебры $W$, назовем центром супералгебры $W$, т.е. $a_{Z} \in W_{Z} \Rightarrow\left[a_{Z}, b\right]=0$ для любого $b \in W$. Очевидно, что множество $W_{Z}$ состоит из функций, не зависящих от $y: a_{Z} \in W_{Z} \Rightarrow a_{Z}=a_{Z 0}=a_{Z}(z \mid \eta ; \hbar)$.

Введем операторы

$$
\hat{\delta}=\eta^{A} \frac{\partial}{\partial y^{A}}, \quad \hat{\delta}^{*}=y^{A} \frac{\partial}{\partial \eta^{A}}, \quad \widehat{N}=\eta^{A} \frac{\partial}{\partial \eta^{A}}+y^{A} \frac{\partial}{\partial y^{A}}, \quad \hat{q}=\widehat{N}^{-1} \cdot \hat{\delta}^{*},
$$

обладающие свойствами

$$
\hat{\delta}^{2}=\left(\hat{\delta}^{*}\right)^{2}=\hat{q}^{2}=0, \quad \hat{\delta} \hat{\delta}^{*}+\hat{\delta}^{*} \hat{\delta}=\widehat{N}, \quad[\widehat{N}, \hat{\delta}]=\left[\widehat{N}, \hat{\delta}^{*}\right]=[\widehat{N}, \hat{q}]=0
$$




$$
\begin{gathered}
\varepsilon(\hat{\delta})=\varepsilon\left(\hat{\delta}^{*}\right)=\varepsilon(\hat{q})=1, \quad \varepsilon(\widehat{N})=0, \quad \hat{q} a= \begin{cases}\hat{\delta}^{*} \widehat{N}^{-1} a, & \widehat{N} a \neq 0, \\
0, & \widehat{N} a=0,\end{cases} \\
\operatorname{deg}(\hat{\delta})=-1, \quad \operatorname{deg}\left(\hat{\delta}^{*}\right)=\operatorname{deg}(\hat{q})=1, \quad \operatorname{deg}(\widehat{N})=0, \\
a=\hat{\delta} \hat{\delta}^{*} a+\hat{\delta}^{*} \hat{\delta} a+a_{00} \quad \forall a \in W .
\end{gathered}
$$

Введем матрицу $\omega_{A B}(z)$, обратную матрице $\omega^{A B}(z)$,

$$
\begin{gathered}
\omega_{A D}(z) \omega^{D B}(z)=\omega^{B D}(z) \omega_{D A}(z)=\delta_{A}^{B}, \\
\omega_{B A}=(-1)^{\left(1+\varepsilon_{A}\right)\left(1+\varepsilon_{B}\right)} \omega_{A B}, \quad \varepsilon\left(\omega_{A B}\right)=\varepsilon_{A}+\varepsilon_{B}, \\
(-1)^{\left(1+\varepsilon_{A}\right) \varepsilon_{C}} \partial_{A}^{z} \omega_{B C}(z)+\operatorname{cycle}(A, B, C)=0 .
\end{gathered}
$$

Ковариантными производными матриц $\omega_{A B}(z)$ и $\omega^{A B}(z)$ назовем выражения

$$
\begin{aligned}
\nabla_{A} \omega_{B C}(z) & =\partial_{A}^{z} \omega_{B C}(z)-\Delta_{A B}^{D}(z) \omega_{D C}(z)-(-1)^{\left(1+\varepsilon_{B}\right)\left(1+\varepsilon_{C}\right)} \Delta_{A C}^{D}(z) \omega_{D B}(z), \\
\nabla_{A} \omega^{B C}(z) & =(-1)^{\varepsilon_{B}\left(\varepsilon_{A}+\varepsilon_{D}\right)} \omega^{B D}(z)\left[\nabla_{A} \omega_{D F}(z)\right] \omega^{F C}(z)= \\
& =\partial_{A}^{z} \omega^{B C}(z)+\Gamma_{A D}^{B}(z) \omega^{D C}(z)-(-1)^{\varepsilon_{B} \varepsilon_{C}} \Gamma_{A D}^{C}(z) \omega^{D B}(z), \\
\Delta_{B A}^{C} & =(-1)^{\varepsilon_{A} \varepsilon_{B}} \Delta_{A B}^{C}, \quad \varepsilon\left(\Delta_{A B}^{C}\right)=\varepsilon_{A}+\varepsilon_{B}+\varepsilon_{C}, \quad \Gamma_{A B}^{C}=(-1)^{\varepsilon_{B}\left(1+\varepsilon_{C}\right)} \Delta_{A B}^{C},
\end{aligned}
$$

где $\Delta_{A B}^{C}(z)$ и $\Gamma_{A B}^{C}(z)-$ связности.

Связность называется суперсимплектической, если матрицы $\omega_{A B}(z)$ и $\omega^{A B}(z)$ (суперсимплектические метрики) ковариантно постоянны, т.е.

$$
\nabla_{A} \omega_{B C}(z)=\nabla_{A} \omega^{B C}(z)=0 .
$$

Уравнение (6.9) можно записать в виде

$$
\partial_{A}^{z} \omega_{B C}(z)=\Delta_{A B \mid C}(z)-(-1)^{\varepsilon_{B} \varepsilon_{C}} \Delta_{A C \mid B}(z), \quad \Delta_{A B \mid C}=\Delta_{A B}^{D} \omega_{D C}(-1)^{\varepsilon_{C}},
$$

следствием которого является полезное соотношение

$$
\begin{aligned}
& {\left[\partial_{A}^{z} \omega_{B C}(z)-(-1)^{\varepsilon_{A} \varepsilon_{B}} \partial_{B}^{z} \omega_{A C}(z)\right]=} \\
& \quad=-(-1)^{\varepsilon_{B} \varepsilon_{C}} \Delta_{A C \mid B}(z)+(-1)^{\varepsilon_{A}\left(\varepsilon_{B} \varepsilon_{C}\right)} \Delta_{B C \mid A}(z) .
\end{aligned}
$$

Общее решение уравнения (6.10) можно представить в виде

$$
\Delta_{A B \mid C}(z)=\frac{1}{3}\left[\partial_{A}^{z} \omega_{B C}(z)+\partial_{B}^{z} \omega_{A C}(z)\right](-1)^{\varepsilon_{C}}+\rho_{A B C}(z),
$$

где $\rho_{A B C}(z)$ - произвольная функция, полностью суперсимметричная по индексам.

Введем следующие операторы: дифференциал $\hat{d}=\eta^{A} \partial_{A}^{z}$, операторная связность $\widehat{\Gamma}=\eta^{A} \Gamma_{A B}^{C} y^{B} \partial_{C}^{z}=\eta^{A} \Delta_{A B}^{C}(-1)^{\varepsilon_{B}\left(1+\varepsilon_{C}\right)} y^{B} \partial_{C}^{z}$, операторная ковариантная производная $\widehat{D}=\hat{d}-\widehat{\Gamma} \equiv \eta^{A} \widehat{D}_{A}$.

Прямое вычисление дает следующее правило действия операторной ковариантной производной на произведение Вейля:

$$
\begin{aligned}
\widehat{D}(a \circ b) & =(\widehat{D} a) \circ b+(-1)^{\varepsilon(a)} a \circ(\widehat{D} b)+\frac{i \hbar}{2}(-1)^{\xi} \eta^{A}\left[\nabla_{A} \omega^{B C}\right]\left(a \overleftarrow{\partial}_{B}^{y}\right) \circ\left(\partial_{C}^{y} b\right), \\
\xi & =\left(\varepsilon(a)+\varepsilon_{C}+1\right) \varepsilon_{B}+\varepsilon(a) \varepsilon_{C} .
\end{aligned}
$$


Если связность суперсимплектическая, то операторная ковариантная производная действует на произведение Вейля по правилу Лейбница

$$
\widehat{D}(a \circ b)=(\widehat{D} a) \circ b+(-1)^{\varepsilon(a)} a \circ(\widehat{D} b) .
$$

Далее мы всегда будем предполагать, что связность суперсимплектическая.

Непосредственное вычисление позволяет получить следующие соотношения:

$$
\begin{aligned}
\widehat{D}^{2} a & =\frac{1}{2}(-1)^{\varepsilon_{B}} \eta^{B} \eta^{A}\left[\widehat{D}_{A}, \widehat{D}_{B}\right] a= \\
& =\frac{1}{2}(-1)^{\varepsilon_{B}} \eta^{B} \eta^{A} R_{A B \mid C}{ }^{D} y^{C}(-1)^{\varepsilon_{C}\left(1+\varepsilon_{D}\right)} \partial_{D}^{y} a=-\frac{i}{\hbar}[R, a], \\
\widehat{D} R & =0, \quad R=\frac{1}{4} R_{A B} y^{A} y^{B}, \\
R_{A B} & =(-1)^{\varepsilon_{D}} \eta^{D} \eta^{C} R_{C D|A| B}, \quad R_{C D|A| B}=R_{C D \mid A}{ }^{D} \omega_{D B}(-1)^{\varepsilon_{C}\left(1+\varepsilon_{D}\right)},
\end{aligned}
$$

где $R_{A B \mid C}{ }^{D}=-\partial_{A}^{z} \Delta_{B C}^{D}+\Delta_{A C}^{E} \Delta_{E B}^{D}(-1)^{\varepsilon_{B} \varepsilon_{C}}-(-1)^{\varepsilon_{A} \varepsilon_{B}}(A \leftrightarrow B)-$ тензор кривизны связности $\Delta_{A B}^{C}$. Используя соотношения $(6.8),(6.10)$ и $(6.11)$, получаем представление для тензора $R_{A B|C| D}$ :

$$
\begin{aligned}
R_{A B|C| D}= & \frac{1}{3} \partial_{C}^{z} \partial_{D}^{z} \omega_{A B}(-1)^{\varepsilon_{B}+\left(\varepsilon_{A}+\varepsilon_{B}\right)\left(\varepsilon_{C}+\varepsilon_{D}\right)}+\left[\partial_{A} \rho_{B C D}-(-1)^{\varepsilon_{A} \varepsilon_{B}} \partial_{B} \rho_{A C D}\right]+ \\
& +\left[\Delta_{A C \mid E} \omega^{E F} \Delta_{B D \mid F}(-1)^{\varepsilon_{C}+\varepsilon_{D}+\varepsilon_{D C}+\varepsilon_{E}+\varepsilon_{B} \varepsilon_{C}+\varepsilon_{B} \varepsilon_{F}+\varepsilon_{D} \varepsilon_{F}}-\right. \\
& \left.-(-1)^{\varepsilon_{A} \varepsilon_{B}}(A \leftrightarrow B)\right],
\end{aligned}
$$

явно демонстрирующее суперантисимметрию этого тензора по индексам $A, B$ и его суперсимметрию по индексам $C, D$.

Простые вычисления позволяют установить следующие соотношения:

$$
\hat{\delta} \widehat{D}+\widehat{D} \hat{\delta}=0, \quad \hat{\delta} a=-\frac{i}{\hbar}\left[\gamma_{\delta}, a\right], \quad \gamma_{\delta}=\eta^{A} \omega_{A B}(z) y^{B}
$$

Оператор $\widehat{Q}$,

$$
\widehat{Q} a=\widehat{D} a-\frac{i}{\hbar}[\gamma, a]
$$

назовем $\widehat{Q}$-связностью, а функцию (2-форму) $\Omega$,

$$
\Omega=R+\widehat{D} \gamma-\frac{i}{\hbar} \gamma \circ \gamma
$$

- ее кривизной. Здесь $\gamma=\eta^{A} \gamma_{A}(z \mid y ; \hbar)$ - пока произвольная функция первого порядка по $\eta$ (1-форма) со свойством $\gamma_{0}=0$. $\widehat{Q}$-связность обладает следующими свойствами (напомним, что операторная ковариантная производная $\widehat{D}$ суперсимплектическая):

$$
\begin{gathered}
\widehat{Q}^{2} a=-\frac{i}{\hbar}[\Omega, a], \quad \widehat{Q} \Omega=0, \\
\widehat{Q}(a \circ b)=(\widehat{Q} a) \circ b+(-1)^{\varepsilon(a)} a \circ(\widehat{Q} b) .
\end{gathered}
$$

$\widehat{Q}$-связность называется абелевой, если $\widehat{Q}^{2} a=0$ для любого $a \in W$, или, эквивалентно, $\Omega=\Omega_{0} \in W_{Z}$. 
Теорема 1. Существует абелева $\widehat{Q}$-связность вида

$$
\widehat{Q} a=\widehat{D} a-\hat{\delta} a-\frac{i}{\hbar}[r, a]=\widehat{D} a-\frac{i}{\hbar}[\gamma, a], \quad \gamma=-\gamma_{\delta}+r
$$

где 1-форма $г$ является (единственным) решением уравнения

$$
\hat{\delta} r=R+\widehat{D} r-\frac{i}{\hbar} r \circ r, \quad \operatorname{deg} r \geqslant 3, \quad \hat{q} r=0 .
$$

Доказательство существования и единственности решения уравнения (6.13) повторяет рассуждения Федосова и мы его здесь не приводим.

Вычисление кривизны $\widehat{Q}$-связности дает

$$
\Omega=\frac{1}{2} \eta^{A} \eta^{B} \omega_{B A}-\hat{\delta} r+R+\widehat{D} r-\frac{i}{\hbar} r \circ r=\frac{1}{2} \eta^{A} \eta^{B} \omega_{B A} .
$$

Далее мы рассматриваем только абелевы $\widehat{Q}$-связности.

Функцию $a \in W$, удовлетворяющую условию

$$
\widehat{Q} a=0,
$$

назовем плоской. Подпространство плоских функций из $W$, не зависящих от $\eta$ (подпространство плоских 0-форм), обозначим $W_{Q}$ :

$$
a_{Q} \in W_{Q} \Rightarrow a_{Q}=a_{Q}(z \mid y ; \hbar), \quad \widehat{Q} a_{Q}=0 .
$$

Подпространство функций из $W_{Z}$, не зависящих от $\eta$, обозначим $W_{Z 0}$ :

$$
a_{Z 0} \in W_{Z 0} \Rightarrow a_{Z 0}=a_{Z 0}(z \mid \hbar) .
$$

Справедлива следующая

ЛЕмма 5. Подпространство $W_{Q}$ является алгеброй относительно произведения Вейля.

Действительно,

$$
\widehat{Q}\left(a_{Q} \circ b_{Q}\right)=\left(\widehat{Q} a_{Q}\right) \circ b_{Q}+(-1)^{\varepsilon(a)} a \circ \widehat{Q} b_{Q}=0 \Rightarrow a_{Q} \circ b_{Q} \in W_{Q} .
$$

Введем оператор $\hat{\sigma}$ перехода в точку $y^{A}=0$ :

$$
\hat{\sigma} a(\ldots y \ldots)=a(\ldots 0 \ldots)=a_{0} .
$$

Следующая теорема показывает, что между пространствами $W_{Q}$ и $W_{Z 0}$ существует естественное взаимно однозначное соответствие.

Теорема 2. Для любой функиии $a_{Z 0} \in W_{Z 0}$ существует одна и только одна бункиия $a_{Q} \in W_{Q}$ со свойством $\hat{\sigma} a_{Q}=a_{Z 0}$. Другими словами, уравнение

$$
\widehat{Q} a=0, \quad a=a(z \mid y ; \hbar), \quad a(z \mid 0 ; \hbar)=a_{Z 0}(z \mid \hbar),
$$

имеет при фиксированной функции $a_{Z 0}(z \mid \hbar)$ единственное решение.

Доказательство повторяет рассуждения Федосова и мы его здесь не приводим. 
Функции $a_{Q} \in W_{Q}, \hat{\sigma} a_{Q}=a_{Z 0} \in W_{Z 0}$, будем называть расширениями функций $a_{Z 0}$.

ОПРЕДЕЛЕНИЕ. Пусть $a_{Z 0}, b_{Z 0} \in W_{Z 0}$, а $a_{Q}, b_{Q} \in W_{Q}$ - соответствующие расширения этих функций. Назовем *-произведением $a_{Z 0} * b_{Z 0}$ функций $a_{Z 0}$ и $b_{Z 0}$ выражение

$$
a_{Z 0} * b_{Z 0}=\hat{\sigma}\left[a_{Q} \circ b_{Q}\right] .
$$

Нетрудно проверить, что *-произведение (6.14) ассоциативно.

Далее мы будем использовать конкретное выражение для связности ${ }^{3)} \Delta_{A B}^{C}$,

$$
\Delta_{A B}^{C}=\frac{1}{3}\left(\partial_{A}^{z} \omega_{B D}+(-1)^{\varepsilon_{A} \varepsilon_{B}} \partial_{B}^{z} \omega_{A D}\right) \omega^{D C},
$$

где $\omega^{A B}$ - суперсимплектическая метрика, задаваемая формальным рядом по $\hbar$,

$$
\omega^{A B}=\omega^{A B}(z \mid \hbar)=\sum_{k=1} \hbar^{k-1} \omega_{k}^{A B}(z)
$$

При фиксированной метрике $\omega^{A B}$ *-произведение (6.14) однозначно определено и задается формальным рядом по $\hbar$ со структурой

$$
a_{Z 0} * b_{Z 0}=a_{Z 0} b_{Z 0}+O(\hbar) \text {. }
$$

Будем обозначать *-произведение (6.14) как $\Pi_{F \omega}(z \mid f, g ; \hbar)$.

6.5. Второй порядок. Представим форму П $(z \mid f, g ; \hbar)$ в виде

$$
\Pi(z \mid f, g ; \hbar)=\Pi_{F \omega_{1}}(z \mid f, g ; \hbar)+\hbar^{2} \Pi_{(2)}(z \mid f, g)+O\left(\hbar^{3}\right), \quad \Pi_{(2)} \in \mathcal{A}_{2} .
$$

Тогда во втором порядке по $\hbar$ уравнение ассоциативности (6.1) принимает вид

$$
\begin{aligned}
d_{2} \Pi_{(2)} & =0 \Rightarrow \\
\Rightarrow \Pi_{(2)}(z \mid f, g) & =m_{\omega_{2}}(z \mid f, g)+d_{1} M_{(2)}(z \mid f, g), \quad M_{(2)}(z \mid f) \in \mathcal{A}_{1} .
\end{aligned}
$$

6.6. Третий порядок. После выполнения преобразования подобия $T=I-$ $\hbar^{2} M_{(2)}(z \mid f)+O\left(\hbar^{2}\right)$ форма П может быть представлена в виде

$$
\begin{aligned}
& \Pi(z \mid f, g ; \hbar)=\Pi_{F \omega_{1}}(z \mid f, g ; \hbar)+\hbar^{2} m_{\omega_{2}}(z \mid f, g)+\hbar^{3} \Pi_{(3)}(z \mid f, g)+O\left(\hbar^{4}\right), \\
& \Pi_{(3)}(z \mid f, g) \in D .
\end{aligned}
$$

Согласно лемме 3 уравнение ассоциативности (6.1) дает в третьем порядке по $\hbar$

$$
d_{3} \Pi_{(3)}+\operatorname{Asc}\left(m_{\omega_{1}}, m_{\omega_{2}}\right)=0 .
$$

\footnotetext{
3) Выражение (6.15), рассматриваемое в произвольной системе координат, не имеет трансформационных свойств связности. Однако его легко ковариантизовать таким образом, что в произвольной системе координат это будет настоящая связность, а в используемой нами (декартовой) системе координат она будет совпадать с выражением (6.15).
} 
Используя тождество (6.3), получаем

$$
\begin{gathered}
(-1)^{\varepsilon(f) \varepsilon(h)}\left[m_{\omega_{1}}\left(z \mid f, m_{\omega_{2}}(\mid g, h)\right)+m_{\omega_{2}}\left(z \mid f, m_{\omega_{1}}(\mid g, h)\right)\right]+\operatorname{cycle}(f, g, h)=0 \Rightarrow \\
\Rightarrow(-1)^{\varepsilon_{A} \varepsilon_{C}}\left[\omega_{1}^{A D}(z) \partial_{D} \omega_{1}^{B C}(z)+\omega_{2}^{A D}(z) \partial_{D} \omega_{1}^{B C}(z)\right]+\operatorname{cycle}(f, g, h)=0 .
\end{gathered}
$$

Соотношение (6.16) означает, что метрика $\omega_{1}^{A B}+\hbar \omega_{2}^{A B}$ удовлетворяет тождеству Якоби (6.7) с точностью до членов порядка $\hbar^{2}$. Отсюда следует, что матрица

$$
\omega_{[1] A B}(z ; \hbar)=\omega_{1 A B}(z)+\hbar \omega_{1 C D}(z) \omega_{2}^{C D}(z) \omega_{1 D B}(z)
$$

удовлетворяет тождеству (6.8), а значит, суперантисимметричная матрица $\omega_{[1]}^{A B}(z ; \hbar)$,

$$
\omega_{[1]}^{A C}(z ; \hbar) \omega_{[1] C B}(z ; \hbar)=\delta_{B}^{A}, \quad \omega_{[1]}^{A B}(z ; \hbar)=\omega_{1}^{A B}(z)+\hbar \omega_{2}^{A B}(z)+O\left(\hbar^{2}\right),
$$

точно удовлетворяет тождеству Якоби (6.7).

В силу (6.6) мы теперь можем представить форму $(z \mid f, g ; \hbar)$ в виде

$$
\Pi(z \mid f, g ; \hbar)=\Pi_{F \omega_{[1]}}(z \mid f, g ; \hbar)+\hbar^{3} \Pi_{(3)}(z \mid f, g)+O\left(\hbar^{4}\right), \quad \Pi_{(3)}(z \mid f, g) \in D .
$$

Тогда в третьем порядке по $ћ$ уравнение ассоциативности (6.1) принимает вид

$$
d_{2} \Pi_{(3)}=0
$$

Далее нам остается воспользоваться методом математической индукции. Таким образом, доказана следующая теорема.

ТЕорема 3. Каждая дебормачия $\Pi(z \mid f, g ; \hbar)=f g+\hbar m_{\omega_{1}}(z \mid f, g)+\cdots$ nоточечного произведения, где матрица $\omega_{1}^{A B}(z)$ обратима при всех $z$, эквивалентна с точностью до преобразования подобия звездочному произведению $\Pi_{F \omega_{[\infty]}}(z \mid f, g ; \hbar)$, где

$$
\omega_{[\infty]}^{A B}(z ; \hbar)=\omega_{1}^{A B}(z)+\hbar \omega_{2}^{A B}(z)+\cdots
$$

- суперантисимметричная матрица, удовлетворяющая тождеству Якоби (6.7).

ПРИЛОЖКНИЕ А

\section{Свойства гладкости первого дифференциала Хохшильда}

Пусть $O$ - открытое множество в $\mathbb{R}^{n}$. Обозначим через $\mathcal{E}(O)$ пространство всех гладких функций на $O$. Топология $\mathcal{E}(O)$ задается полунормами

$$
\|f\|_{N, K}=\sup _{x \in K,|\lambda| \leqslant N}\left|\partial^{\lambda} f(x)\right|
$$

где $N$ пробегает множество $\mathbb{Z}_{+}$неотрицательных целых чисел, а $K$ - множество всех компактов, лежащих в $O$.

Пусть $K$ - компакт в $\mathbb{R}^{n}$. Подпространство $\mathcal{E}\left(\mathbb{R}^{n}\right)$, состоящее из функций, носитель которых содержится в $K$, обозначим $\mathcal{D}(K)$. Пространство $\mathcal{D}(K)$ снабжается топологией, индуцированной из $\mathcal{E}\left(\mathbb{R}^{n}\right)$. Если $V$ - открытое множество в $\mathbb{R}^{n}$, то пространство Шварца $\mathcal{D}(V)$ определяется соотношением $\mathcal{D}(V)=\bigcup_{K} \mathcal{D}(K)$, где 
объединение берется по всем компактам, лежащим в $V$, и снабжается топологией индуктивного предела.

Пусть $O$ и $V$ - открытые множества в $\mathbb{R}^{n_{1}}$ и $\mathbb{R}^{n_{2}}$ соответственно, $\mathbf{m}$ - непрерывное линейное отображение из $\mathcal{D}(V)$ в $\mathcal{E}(O)$.

Очевидно, билинейная форма

$$
(f, g) \rightarrow \int f(x) \mathbf{m}(g)(x) d x
$$

на $\mathcal{D}(O) \times \mathcal{D}(V)$ раздельно непрерывна. Поэтому в силу теоремы Шварца о ядpe (см. [17], теорема 5.2.1) существует единственная обобщенная функция $m \in$ $\mathcal{D}^{\prime}(O \times V)$ такая, что

$$
m(f \otimes g)=\int f(x) \mathbf{m}(g)(x) d x, \quad(f \otimes g)(x, y)=f(x) g(y),
$$

для любых $f \in \mathcal{D}(O)$ и $g \in \mathcal{D}(V)$. Мы будем говорить, что обобщенная функция $m \in \mathcal{D}^{\prime}(O \times V)$ является гладкой по первому аргументу, если она может быть получена описанным выше способом, т.е. существует непрерывное линейное отображение $\mathbf{m}: \mathcal{D}(V) \rightarrow \mathcal{E}(O)$, удовлетворяющее (А.1).

Лемма 6. Пусть $O \subset \mathbb{R}^{n_{1}}$ - открытое множество, $\varrho$ - гладкое отображение из $O$ в $\mathbb{R}^{n_{2}}$. Если обобщенная функция $m \in \mathcal{D}^{\prime}\left(O \times \mathbb{R}^{n_{2}}\right)$ гладкая по первому аргументу, то обобщенная функиия $m(x, y+\varrho(x))$ также является гладкой по первому аргументу.

Доказательство леммы 6 будет дано ниже в приложении Б.

Первый дифференциал Хохшильда $d_{1}$ будем рассматривать как оператор из пространства $\mathcal{D}^{\prime}\left(\mathbb{R}^{2 n}\right)$ в $\mathcal{D}^{\prime}\left(\mathbb{R}^{3 n}\right)$. Для любого $M \in \mathcal{D}^{\prime}\left(\mathbb{R}^{2 n}\right)$ имеем

$$
d_{1} M(\varphi)=M(\mathcal{L} \varphi), \quad \varphi \in \mathcal{D}\left(\mathbb{R}^{3 n}\right)
$$

где непрерывный линейный оператор $\mathcal{L}: \mathcal{D}\left(\mathbb{R}^{3 n}\right) \rightarrow \mathcal{D}\left(\mathbb{R}^{2 n}\right)$ определяется соотношением

$$
(\mathcal{L} \varphi)(x, y)=\varphi(x, x, y)+\varphi(x, y, x)-\varphi(x, y, y) .
$$

Фиксируем $M \in \mathcal{D}^{\prime}\left(\mathbb{R}^{2 n}\right)$ и введем обобщенные функции $\widetilde{M} \in \mathcal{D}^{\prime}\left(\mathbb{R}^{2 n}\right)$ и $S \in \mathcal{D}^{\prime}\left(\mathbb{R}^{3 n}\right)$, полагая

$$
\widetilde{M}(x, y)=M(x, y+x), \quad S(x, y, z)=d_{1} M(x, y+x, z+x) .
$$

Из (А.2) и (А.3) следует, что $\widetilde{M}$ и $S$ связаны соотношением

$$
S(\varphi)=\widetilde{M}(\widetilde{\mathcal{L}} \varphi), \quad \varphi \in \mathcal{D}\left(\mathbb{R}^{3 n}\right),
$$

где непрерывный линейный оператор $\widetilde{\mathcal{L}}: \mathcal{D}\left(\mathbb{R}^{3 n}\right) \rightarrow \mathcal{D}\left(\mathbb{R}^{2 n}\right)$ дается формулой

$$
(\widetilde{\mathcal{L}} \varphi)(x, y)=\varphi(x, 0, y)+\varphi(x, y, 0)-\varphi(x, y, y)
$$

В частности, если $f \in \mathcal{D}\left(\mathbb{R}^{n}\right)$ и $\psi \in \mathcal{D}\left(\mathbb{R}^{2 n}\right)$, то

$$
S(f \otimes \psi)=\widetilde{M}(f \otimes Q \psi),
$$

где непрерывный линейный оператор $Q: \mathcal{D}\left(\mathbb{R}^{2 n}\right) \rightarrow \mathcal{D}\left(\mathbb{R}^{n}\right)$ имеет вид

$$
(Q \psi)(x)=\psi(0, x)+\psi(x, 0)-\psi(x, x) .
$$


Лемма 7. Для любого компакта $K \subset \mathbb{R}^{n}$ существует такой непрерывный линейный оператор $R_{K}: \mathcal{D}\left(\mathbb{R}^{n}\right) \rightarrow \mathcal{D}\left(\mathbb{R}^{2 n}\right)$, что

$$
Q R_{K} g=g
$$

для любой функиии $g \in \mathcal{D}(K)$ такой, что

$$
\partial_{i} g(0)=0, \quad i=1,2, \ldots, n .
$$

ДоказАтельство. Пусть $K$ - компакт в $\mathbb{R}^{n}, h_{K}-$ гладкая функция с компактным носителем, равная единице в окрестности $K$ и удовлетворяющая условию $h_{K}(0)=1$. Определим оператор $R_{K}$ соотношением

$$
\left(R_{K} g\right)(x, y)=g(x) h_{K}(y)^{2}-h_{K}(x) h_{K}(y) \sum_{i=1}^{n} y^{i} \int_{0}^{1}\left(\partial_{i} g\right)(t x) d t, \quad g \in \mathcal{D}\left(\mathbb{R}^{n}\right) .
$$

Непосредственно проверяется, что оператор $R_{K}$ непрерывен. Если $g \in \mathcal{D}(K)$ удовлетворяет (А.9), то интегралы $\int_{0}^{1}\left(\partial_{i} g\right)(t x) d t$ обращаются в нуль при $x=0$. Поэтому в силу (А.7) и соотношения $h_{K}^{2} g=g$ при любом $x \in \mathbb{R}^{n}$ имеем

$$
\begin{aligned}
\left(Q R_{K} g\right)(x) & =g(0) h_{K}(x)^{2}+g(x)\left(1-h_{K}(x)^{2}\right)+h_{K}(x)^{2} \sum_{i=1}^{n} x^{i} \int_{0}^{1}\left(\partial_{i} g\right)(t x) d t= \\
& =h_{K}(x)^{2}\left(g(0)+\int_{0}^{1} \frac{d}{d t} g(t x) d t\right)=h_{K}(x)^{2} g(x)=g(x) .
\end{aligned}
$$

Пусть $\chi$ - гладкая функция с компактным носителем на $\mathbb{R}^{n}$, равная единице в окрестности начала координат. Для $g \in \mathcal{D}\left(\mathbb{R}^{n}\right)$ определим функцию $\hat{g} \in \mathcal{D}\left(\mathbb{R}^{n}\right)$, полагая

$$
\hat{g}(x)=g(x)-\chi(x) \sum_{i=1}^{n}\left(\partial_{i} g\right)(0) x^{i} .
$$

Очевидно, $\left(\partial_{i} \hat{g}\right)(0)=0$ для любого $i=1,2, \ldots, n$.

Предположим, что обобщенная функция $d_{1} M$ является гладкой по первому аргументу. Тогда в силу леммы 6 обобщенная функция $S$ также является гладкой по первому аргументу. По определению это означает, что существует такое непрерывное линейное отображение $\mathbf{S}: \mathcal{D}\left(\mathbb{R}^{2 n}\right) \rightarrow \mathcal{E}\left(\mathbb{R}^{n}\right)$, что

$$
S(f \otimes \psi)=\int \mathbf{S}(\psi)(x) f(x) d x, \quad f \in \mathcal{D}\left(\mathbb{R}^{n}\right), \quad \psi \in \mathcal{D}\left(\mathbb{R}^{2 n}\right) .
$$

Для каждого компакта $K \subset \mathbb{R}^{n}$ введем линейное отображение $\widetilde{\mathbf{m}}_{K}: \mathcal{D}(K) \rightarrow \mathcal{D}\left(\mathbb{R}^{n}\right)$, полагая

$$
\widetilde{\mathbf{m}}_{K}(g)=\mathbf{S}\left(R_{K} \hat{g}\right), \quad g \in \mathcal{D}(K)
$$

где $R_{K}$ - оператор, удовлетворяющий условиям леммы 7 . Поскольку $g \rightarrow \hat{g}$ есть непрерывное отображение из $\mathcal{D}(K)$ в $\mathcal{D}\left(\mathbb{R}^{n}\right)$, отображения $\widetilde{\mathbf{m}}_{K}$ непрерывны для 
всех $K$. Если $K \supset \operatorname{supp} \chi$ и $g \in \mathcal{D}(K)$, то $\hat{g} \in \mathcal{D}(K)$. Поэтому при $K \supset \operatorname{supp} \chi$ в силу (А.6), (А.11) и леммы 7 имеем

$$
\begin{aligned}
\int \widetilde{\mathbf{m}}_{K}(g)(x) f(x) d x & =\int \mathbf{S}\left(R_{K} \hat{g}\right)(x) f(x) d x= \\
& =S\left(f \otimes R_{K} \hat{g}\right)=\widetilde{M}\left(f \otimes Q R_{K} \hat{g}\right)= \\
& =\widetilde{M}(f \otimes \hat{g}), \quad f \in \mathcal{D}\left(\mathbb{R}^{n}\right), \quad g \in \mathcal{D}(K) .
\end{aligned}
$$

Поскольку правая часть (А.12) не зависит от $K$, имеем $\widetilde{\mathbf{m}}_{K^{\prime}}(g)=\widetilde{\mathbf{m}}_{K}(g)$ для любых компактов $K^{\prime} \supset K \supset \operatorname{supp} \chi$ и любой функции $g \in \mathcal{D}(K)$. Это означает, что отображения $\widetilde{\mathbf{m}}_{K}$ при разных $K$, содержащих $\operatorname{supp} \chi$, согласованы между собой и, следовательно, определяют непрерывное линейное отображение $\widetilde{\mathbf{m}}: \mathcal{D}\left(\mathbb{R}^{n}\right) \rightarrow \mathcal{E}\left(\mathbb{R}^{n}\right)$. В силу (А.12) имеем

$$
\int \widetilde{\mathbf{m}}(g)(x) f(x) d x=\widetilde{M}(f \otimes \hat{g}), \quad f, g \in \mathcal{D}\left(\mathbb{R}^{n}\right) .
$$

Пусть $\widetilde{m}$ - гладкая обобщенная функция по первому аргументу, соответствующая отображению $\widetilde{\mathbf{m}}$, т.е. такая обобщенная функция на $\mathbb{R}^{2 n}$, что

$$
\widetilde{m}(f \otimes g)=\int \widetilde{\mathbf{m}}(g)(x) f(x) d x, \quad f, g \in \mathcal{D}\left(\mathbb{R}^{n}\right) .
$$

Для $i=1,2, \ldots, n$ положим $\chi^{i}(x)=\chi(x) x^{i}$ и определим обобщенные функции $\mu^{i} \in$ $\mathcal{D}^{\prime}\left(\mathbb{R}^{n}\right)$ соотношениями $\mu^{i}(f)=-\widetilde{M}\left(f \otimes \chi^{i}\right), f \in \mathcal{D}\left(\mathbb{R}^{n}\right)$. Тогда в силу (А.10) и (А.13) получаем

$$
\widetilde{m}(f \otimes g)=\widetilde{M}(f \otimes g)+\sum_{i=1}^{n}\left(\partial_{i} g\right)(0) \mu^{i}(f), \quad f, g \in \mathcal{D}\left(\mathbb{R}^{n}\right) .
$$

Поскольку линейная оболочка элементов вида $f \otimes g$ плотна в $\mathcal{D}\left(\mathbb{R}^{2 n}\right)$, из (А.14) следует, что

$$
\widetilde{m}(x, y)=\widetilde{M}(x, y)-\sum_{i=1}^{n} \mu^{i}(x) \partial_{i} \delta(y) .
$$

Определим обобщенную функцию $m \in \mathcal{D}^{\prime}\left(\mathbb{R}^{2 n}\right)$ формулой $m(x, y)=\widetilde{m}(x, y-x)$. В силу леммы 6 обобщенная функция $m$ является гладкой по первому аргументу. Делая в (А.15) замену $y \rightarrow y-x$, с учетом (А.4) получаем

$$
m(x, y)=M(x, y)-\sum_{i=1}^{n} \mu^{i}(x) \partial_{i} \delta(y-x) .
$$

Таким образом, нами доказано следующее утверждение.

Лемма 8. Пусть $M \in \mathcal{D}^{\prime}\left(\mathbb{R}^{2 n}\right)$ - обобщенная функиия такая, что $d_{1} M$ является гладкой по первому аргументу. Тогда $M$ представима в виде

$$
M(x, y)=m(x, y)+\sum_{i=1}^{n} \mu^{i}(x) \partial_{i} \delta(y-x)
$$

где $\mu^{i} \in \mathcal{D}^{\prime}\left(\mathbb{R}^{n}\right)$, а функция $m \in \mathcal{D}^{\prime}\left(\mathbb{R}^{2 n}\right)$ является гладкой по первому аргументу. 


\section{Доказательство леммы 6}

ПРИЛОЖКНИЕ Б

Пусть $U$ и $V$ - открытые множества в $\mathbb{R}^{n_{1}}$ и $\mathbb{R}^{n_{2}}$ соответственно, $f$ - гладкая функция на $U \times V$. Для $x \in U$ введем функцию $f_{x} \in \mathcal{E}(V)$, полагая

$$
f_{x}(y)=f(x, y) .
$$

Если $\lambda \in \mathbb{Z}_{+}^{n_{1}}$, то функцию $\partial_{x}^{\lambda} f(x, y)$ на $U \times V$ обозначим через $f^{\lambda}$. Для краткости будем писать $f_{x}^{\lambda}$ вместо $\left(f^{\lambda}\right)_{x}$. Таким образом, имеем

$$
f_{x}^{\lambda}(y)=\partial_{x}^{\lambda} f(x, y)
$$

Обозначим через $\mathcal{R}(U, V)$ пространство, состоящее из всех гладких функций $f$ на $U \times V$, обладающих следующим свойством: для любого компактного множества $B \subset U$ найдется такой компакт $K \subset V$, что $f(x, y)=0$ для любых $x \in B$ и $y \notin K$. Очевидно, $f^{\lambda} \in \mathcal{R}(U, V)$ для любых $f \in \mathcal{R}(U, V)$ и $\lambda \in \mathbb{Z}_{+}^{n_{1}}$. Если $f \in \mathcal{R}(U, V)$ и $x \in U$, то $f_{x} \in \mathcal{D}(V)$.

Следующее утверждение непосредственно следует из теоремы 2.1.3 книги [17].

Лемма 9. Пусть $U$ и $V$ - открытые множества в $\mathbb{R}^{n_{1}} u \mathbb{R}^{n_{2}}$ соответственно, $f \in \mathcal{R}(U, V)$ и $m \in \mathcal{D}^{\prime}(V)$. Обозначим через $g$ функцию $x \rightarrow m\left(f_{x}\right)$ на $U$. Тогда $g \in \mathcal{E}(U)$, причем для любых $x \in U$ и мультииндекса $\lambda \in \mathbb{Z}_{+}^{n_{1}}$ имеем

$$
\partial^{\lambda} g(x)=m\left(f_{x}^{\lambda}\right)
$$

Лемма 10. Пусть $U, V$ и $O$-открытые множества в $\mathbb{R}^{n_{1}}, \mathbb{R}^{n_{2}}$ u $\mathbb{R}^{n_{3}}$ соответственно и $f \in \mathcal{R}(U, V)$. Для любого непрерывного отображения $\mathbf{m}: \mathcal{D}(V) \rightarrow \mathcal{E}(O)$ и любого мультииндекса $\nu \in \mathbb{Z}_{+}^{n_{3}}$ функция $(x, z) \rightarrow\left(\partial^{\nu} \mathbf{m}\left(f_{x}\right)\right)(z)$ непреръвна на $U \times O$.

ДоказАтельСтво. Обозначим через $g(x, z)$ функцию $(x, z) \rightarrow\left(\partial^{\nu} \mathbf{m}\left(f_{x}\right)\right)(z)$ на $U \times O$. Фиксируем точку $\left(x_{0}, z_{0}\right) \in U \times O$ и $\varepsilon>0$. Так как функция $\partial^{\nu} \mathbf{m}\left(f_{x_{0}}\right)$ непрерывна на $O$, то найдется такая компактная окрестность $K$ точки $z_{0}$, что

$$
\left|g\left(x_{0}, z\right)-g\left(x_{0}, z_{0}\right)\right|<\frac{\varepsilon}{2}, \quad z \in K .
$$

Легко видеть, что $x \rightarrow f_{x}$ есть непрерывное отображение из $U$ в $\mathcal{D}(V)$. Поэтому найдется такая окрестность $B$ точки $x_{0}$, что $\left\|\mathbf{m}\left(f_{x}\right)-\mathbf{m}\left(f_{x_{0}}\right)\right\|_{|\nu|, K}<\varepsilon / 2$ при $x \in B$. Отсюда, в частности, следует, что $\left|g(x, z)-g\left(x_{0}, z\right)\right|<\varepsilon / 2$ для любых $x \in B$ и $z \in K$. Поэтому в силу (Б.1) имеем $\left|g(x, z)-g\left(x_{0}, z_{0}\right)\right|<\varepsilon$ для любых $x \in B$ и $z \in K$, т.е. $g$ непрерывна в точке $\left(x_{0}, z_{0}\right)$.

Лемма 11. Пусть $U, V$ и $O$-открытые множества в $\mathbb{R}^{n_{1}}, \mathbb{R}^{n_{2}} u \mathbb{R}^{n_{3}}$ соответственно, $f \in \mathcal{R}(U, V)$ u $\mathbf{m}: \mathcal{D}(V) \rightarrow \mathcal{E}(O)$ - непрерывное отображсение. Обозначим через $g$ функцию $(x, z) \rightarrow \mathbf{m}\left(f_{x}\right)(z)$ на $U \times O$. Тогда $g \in \mathcal{E}(U \times O)$, причем

$$
\partial_{x}^{\lambda} \partial_{z}^{\nu} g(x, z)=\left(\partial^{\nu} \mathbf{m}\left(f_{x}^{\lambda}\right)\right)(z)
$$

для любых мультииндексов $\lambda \in \mathbb{Z}_{+}^{n_{1}} u \nu \in \mathbb{Z}_{+}^{n_{3}}$. 
ДокАЗАтельство. Обозначим для краткости $\partial_{x, i}=\partial / \partial x^{i}$ и $\partial_{z, j}=\partial / \partial z^{j}$. Достаточно показать, что для каждого $k \in \mathbb{Z}_{+}$функция $g \in C^{k}(U \times O)$, причем при $|\lambda|+|\nu| \leqslant k$ справедливо равенство (Б.2). Доказательство проведем индукцией по $k$. При $k=0$ утверждение следует из леммы 10. Покажем, что если утверждение верно для $k \in \mathbb{Z}_{+}$, то оно верно и для $k+1$. Пусть $\lambda$ и $\nu$ таковы, что $|\lambda|+|\nu|=k$. Поскольку $\mathbf{m}\left(f_{x}^{\lambda}\right) \in \mathcal{E}(O)$, из (Б.2) следует, что $\partial_{x}^{\lambda} \partial_{z}^{\nu} g$ имеет все частные производные по $z$ и

$$
\partial_{z, j} \partial_{x}^{\lambda} \partial_{z}^{\nu} g(x, z)=\left(\partial^{\tilde{\nu}} \mathbf{m}\left(f_{x}^{\lambda}\right)\right)(z), \quad \tilde{\nu}=\left(\nu_{1}, \ldots, \nu_{j-1}, \nu_{j}+1, \nu_{j+1}, \ldots, \nu_{n_{3}}\right) .
$$

Для $z \in O$ определим обобщенную функцию $m_{z} \in \mathcal{D}^{\prime}(U)$ соотношением $m_{z}(\varphi)=$ $\left(\partial^{\nu} \mathbf{m}(\varphi)\right)(z), \varphi \in \mathcal{D}(U)$. Тогда $\left(\partial^{\nu} \mathbf{m}\left(f_{x}^{\lambda}\right)\right)(z)=m_{z}\left(f_{x}^{\lambda}\right)$. Для любого $\lambda^{\prime} \in \mathbb{Z}_{+}^{n_{1}}$ имеем $\left(f^{\lambda}\right)_{x}^{\lambda^{\prime}}=f_{x}^{\lambda+\lambda^{\prime}}$. Поэтому из (Б.2) и леммы 9 , примененной к функциям $f^{\lambda}$ и $m_{z}$, следует, что $\partial_{x}^{\lambda} \partial_{z}^{\nu} g$ имеет все частные производные по $x$ и

$$
\partial_{x, i} \partial_{x}^{\lambda} \partial_{z}^{\nu} g(x, z)=\left(\partial^{\nu} \mathbf{m}\left(f_{x}^{\tilde{\lambda}}\right)\right)(z), \quad \tilde{\lambda}=\left(\lambda_{1}, \ldots, \lambda_{i-1}, \lambda_{i}+1, \lambda_{i+1}, \ldots, \lambda_{n_{1}}\right) .
$$

Применяя лемму 10 к $f^{\lambda}$, заключаем, что функция $(x, z) \rightarrow\left(\partial^{\nu} \mathbf{m}\left(f_{x}^{\lambda}\right)\right)(z)$ непрерывна на $U \times O$ для любых $\lambda$ и $\nu$. Поэтому из (Б.3) и (Б.4) вытекает непрерывность всех частных производных функции $\partial_{x}^{\lambda} \partial_{z}^{\nu} g(x, z)$. Следовательно, $g \in C^{k+1}(U \times O)$. Наконец, справедливость (Б.2) для производных порядка $k+1$ непосредственно следует из (Б.3) и (Б.4).

ЛЕмма 12. Пусть $O \subset \mathbb{R}^{n_{1}}$ - открытое множество, $\varrho$ - гладкое отображение из $O$ в $\mathbb{R}^{n_{2}}$. Пусть $\mathbf{m}: \mathcal{D}\left(\mathbb{R}^{n_{2}}\right) \rightarrow \mathcal{E}(O)$ - непрерывное линейное отображение. Для $\varphi \in \mathcal{D}\left(\mathbb{R}^{n_{2}}\right)$ определим функцию $\widetilde{\mathbf{m}}(\varphi)$ на $O$ соотношением $\widetilde{\mathbf{m}}(\varphi)(x)=$ $\mathbf{m}(\varphi(\cdot+\varrho(x)))(x)$. Тогда $\widetilde{\mathbf{m}}(\varphi) \in \mathcal{E}(O)$ для любого $\varphi \in \mathcal{D}\left(\mathbb{R}^{n_{2}}\right)$ и $\widetilde{\mathbf{m}}$ является непрерывным линейным отображением из $\mathcal{D}\left(\mathbb{R}^{n_{2}}\right)$ в $\mathcal{E}(O)$.

ДоказАтельство. Для $\varphi \in \mathcal{D}\left(\mathbb{R}^{n_{2}}\right)$ обозначим функцию $(x, y) \rightarrow \varphi(y+\varrho(x))$ на $O \times \mathbb{R}^{n_{2}}$ через $\widetilde{\varphi}$ и определим функцию $\mathbf{n}(\varphi)$ на $O \times O$ соотношением $\mathbf{n}(\varphi)(x, z)=$ $\mathbf{m}\left(\widetilde{\varphi}_{x}\right)(z)$. Очевидно, что $\widetilde{\varphi} \in \mathcal{R}\left(O, \mathbb{R}^{n_{2}}\right)$ для любого $\varphi \in \mathcal{D}\left(\mathbb{R}^{n_{2}}\right)$. Поэтому из леммы 11 следует, что $\mathbf{n}(\varphi) \in \mathcal{E}(O \times O)$. Так как $\widetilde{\mathbf{m}}(\varphi)(x)=\mathbf{n}(\varphi)(x, x)$, то $\widetilde{\mathbf{m}}(\varphi) \in \mathcal{E}(O)$. Докажем, что отображение $\widetilde{\mathbf{m}}$ непрерывно. Поскольку отображение $f(x, z) \rightarrow f(x, x)$ из $\mathcal{E}(O \times O)$ в $\mathcal{E}(O)$ непрерывно, достаточно проверить непрерывность отображения $\mathbf{n}: \mathcal{D}\left(\mathbb{R}^{n_{2}}\right) \rightarrow \mathcal{E}(O \times O)$. Фиксируем $N \in \mathbb{Z}_{+}$и компакты $K \subset O$ и $K^{\prime} \subset \mathbb{R}^{n_{2}}$. Нам надо показать, что найдутся такие $N^{\prime} \in \mathbb{Z}_{+}$и $C>0$, что

$$
\|\mathbf{n}(\varphi)\|_{N, K \times K} \leqslant C\|\varphi\|_{N^{\prime}, K^{\prime}}, \quad \varphi \in \mathcal{D}\left(K^{\prime}\right) .
$$

Выберем такой компакт $K^{\prime \prime} \subset \mathbb{R}^{n_{2}}$, что $K^{\prime}-\varrho(x) \subset K^{\prime \prime}$ для любого $x \in K$. Поскольку отображение $\mathbf{m}$ непрерывно, найдутся такие $N^{\prime \prime} \in \mathbb{Z}_{+}$и $C^{\prime}>0$, что

$$
\|\mathbf{m}(\psi)\|_{N, K} \leqslant C^{\prime}\|\psi\|_{N^{\prime \prime}, K^{\prime \prime}}, \quad \psi \in \mathcal{D}\left(K^{\prime \prime}\right) .
$$

Согласно лемме 11 для любых мультииндексов $\lambda, \nu \in \mathbb{Z}_{+}^{n_{1}}$ и любой функции $\varphi \in$ $\mathcal{D}\left(\mathbb{R}^{n_{2}}\right)$ имеем $\partial_{x}^{\lambda} \partial_{z}^{\nu} \mathbf{n}(\varphi)(x, z)=\left(\partial^{\nu} \mathbf{m}\left(\widetilde{\varphi}_{x}^{\lambda}\right)\right)(z)$. Если $|\nu| \leqslant N$, то в силу (Б.6) получаем, что

$$
\left|\partial_{x}^{\lambda} \partial_{z}^{\nu} \mathbf{n}(\varphi)(x, z)\right| \leqslant C^{\prime}\left\|\widetilde{\varphi}_{x}^{\lambda}\right\|_{N^{\prime \prime}, K^{\prime \prime}}, \quad x, z \in K,
$$


для любого $\varphi \in \mathcal{D}\left(K^{\prime}\right)$. Индукцией по $|\lambda|$ легко доказать, что функцию $\widetilde{\varphi}_{x}^{\lambda}$ можно представить в виде

$$
\widetilde{\varphi}_{x}^{\lambda}(y)=\partial_{x}^{\lambda} \varphi(y+\varrho(x))=\sum_{\kappa \in \mathbb{Z}_{+}^{n_{1}},|\kappa| \leqslant|\lambda|} h_{\lambda, \kappa}(x)\left(\partial^{\kappa} \varphi\right)(y+\varrho(x)),
$$

где $h_{\lambda, \kappa}-$ не зависящие от $\varphi$ гладкие функции на $O$ (их можно выразить через производные от $\varrho$, но это для нас не существенно). Для $\varphi \in \mathcal{D}\left(K^{\prime}\right)$ и $x \in K$ имеем

$$
\left\|\left(\partial^{\kappa} \varphi\right)(\cdot+\varrho(x))\right\|_{N^{\prime \prime}, K^{\prime \prime}} \leqslant\|\varphi\|_{N^{\prime \prime}+|\kappa|, K^{\prime}} .
$$

Поэтому из (Б.7) и (Б.8) следует, что

$$
\left|\partial_{x}^{\lambda} \partial_{z}^{\nu} \mathbf{n}(\varphi)(x, z)\right| \leqslant C_{\lambda}\|\varphi\|_{N^{\prime \prime}+|\lambda|, K^{\prime}}, \quad x, z \in K, \quad|\nu| \leqslant N
$$

где $C_{\lambda}=n_{1}^{|\lambda|} \max _{|\kappa| \leqslant|\lambda|} \sup _{x \in K}\left|h_{\lambda, \kappa}(x)\right|$. Переходя в последней оценке к точной верхней грани по $x, z, \lambda$ и $\nu$ и полагая $N^{\prime}=N^{\prime \prime}+N$ и $C=\max _{|\lambda| \leqslant N} C_{\lambda}$, получаем (Б.5).

Лемма 13. Пусть О и $V$ - открытые множества в $\mathbb{R}^{n_{1}} u \mathbb{R}^{n_{2}}$ соответственно $u \mathbf{m}$ - непрерывное отображение из $\mathcal{D}(V)$ в $\mathcal{E}(O)$. Для любого $\varphi \in \mathcal{D}(O \times V)$ функиия $x \rightarrow \mathbf{m}\left(\varphi_{x}\right)(x)$ на $O$ принадлежит $\mathcal{D}(O)$. Если обобщенная функиия $m \in \mathcal{D}^{\prime}(O \times V)$ является гладкой по первому аргументу и $\mathbf{m}: \mathcal{D}(V) \rightarrow \mathcal{E}(O)$ - непрерывное линейное отображение, удовлетворяющее (А.1), то

$$
m(\varphi)=\int \mathbf{m}\left(\varphi_{x}\right)(x) d x
$$

для любого $\varphi \in \mathcal{D}(O \times V)$.

Доказательство. Для $\varphi \in \mathcal{D}(O \times V)$ обозначим функцию $x \rightarrow \mathbf{m}\left(\varphi_{x}\right)(x)$ на $O$ через $h_{\varphi}$. Очевидно, $h_{\varphi}$ имеет компактный носитель. Кроме того, из леммы 11 следует, что $h_{\varphi}$ - гладкая функция. Таким образом, $h_{\varphi} \in \mathcal{D}(O)$. Поэтому правая часть (Б.9) корректно определена и задает некоторый линейный функционал $\mu$ на $\mathcal{D}(O \times V)$. Покажем, что функционал $\mu$ непрерывен. Пусть $K$ и $K^{\prime}-$ компакты в $O$ и $V$ соответственно. Поскольку отображение $\mathbf{m}$ непрерывно, найдутся такие $N \in \mathbb{Z}_{+}$и $C>0$, что $|\mathbf{m}(g)(x)| \leqslant C\|g\|_{N, K^{\prime}}$ для любых $g \in \mathcal{D}\left(K^{\prime}\right)$ и $x \in K$. Отсюда следует, что

$$
\left|h_{\varphi}(x)\right| \leqslant C\|\varphi(x, \cdot)\|_{N, K^{\prime}} \leqslant C\|\varphi\|_{N, K \times K^{\prime}}, \quad \varphi \in \mathcal{D}\left(K \times K^{\prime}\right), \quad x \in K .
$$

Поэтому для любого $\varphi \in \mathcal{D}\left(K \times K^{\prime}\right)$ имеем $|\mu(\varphi)| \leqslant C \Lambda(K)\|\varphi\|_{N, K \times K^{\prime}}$, где $\Lambda(K)-$ мера Лебега множества $K$, и непрерывность $\mu$ доказана. Из (А.1) и определения $\mu$ следует, что $m(f \otimes g)=\mu(f \otimes g)$ для любых $f \in \mathcal{D}(O)$ и $g \in \mathcal{D}(V)$. Ввиду плотности линейной оболочки элементов вида $f \otimes g$ в $\mathcal{D}(O \times V)$ и непрерывности $m$ и $\mu$ это означает, что $m=\mu$.

ДОКАЗАТЕЛЬСТво ЛЕмМы 6. Положим $\widetilde{m}(x, y)=m(x, y+\varrho(x))$. По условию, найдется непрерывное отображение $\mathbf{m}: \mathcal{D}(V) \rightarrow \mathcal{E}(O)$, удовлетворяющее (А.1). В силу леммы 12 существует такое непрерывное отображение $\widetilde{\mathbf{m}}: \mathcal{D}\left(\mathbb{R}^{n_{2}}\right) \rightarrow \mathcal{E}(O)$, что 
$\widetilde{\mathbf{m}}(g)(x)=\mathbf{m}(g(\cdot-\varrho(x)))(x)$ для любого $g \in \mathcal{D}\left(\mathbb{R}^{n_{2}}\right)$. Пусть $f \in \mathcal{D}(O)$ и $g \in \mathcal{D}\left(\mathbb{R}^{n_{2}}\right)$. Положим $\varphi(x, y)=f(x) g(y-\varrho(x))$. Согласно лемме 13 находим

$$
\widetilde{m}(f \otimes g)=m(\varphi)=\int \mathbf{m}\left(\varphi_{x}\right)(x) d x=\int \widetilde{\mathbf{m}}(g)(x) f(x) d x .
$$

Это означает, что $\widetilde{m}$ является гладкой по первому аргументу.

Благодарности. И.В. Тютин благодарит Институт физики университета СанПаулу за гостеприимство и FAPESP за поддержку. Работа поддержана РФФИ (грант № 08-02-01118 (С. Е. Конштейн, И. В. Тютин)) и Программой поддержки ведущих научных школ (грант НШ-1615.2008.2).

\section{Список литературы}

[1] F. Bayen, M. Flato, C. Fronsdal, A. Lichnerowicz, D. Sternheimer, Ann. Phys., 111:1 (1978), 61-110; 111-151.

[2] М. В. Карасев, В. П. Маслов, Нелинейные скобки Пуассона. Геометрия и квантование, Наука, М., 1991.

[3] B. Fedosov, Deformation Quantization and Index Theory, Math. Top., 9, Akademie, Berlin, 1996.

[4] M. Kontsevich, Lett. Math. Phys., 66:3 (2003), 157-216; arXiv: q-alg/9709040.

[5] N.A. Nekrasov, Trieste lectures on solitons in noncommutative gauge theories, arXiv: hep-th/0011095.

[6] A. Schwarz, Gauge theories on noncommutative spaces, arXiv: hep-th/0011261.

[7] H. J. Groenewold, Physica, 12:7 (1946), 405-460.

[8] Ф. А. Березин, УФН, 132:3 (1980), 497-548.

[9] И. В. Тютин, ТМФ, 127:2 (2001), 253-267; arXiv: hep-th/0101046.

[10] Д. А. Лейтес, И. М. Щепочкина, ТМФ, 126:3 (2001), 339-369.

[11] S.E. Konstein, I. V. Tyutin, General form of the deformation of Poisson superbracket on (2,2)-dimensional superspace, arXiv: hep-th/0612006.

[12] J. Vey, Comment. Math. Helv., 50:1 (1975), 421-454.

[13] G. Pinczon, Lett. Math. Phys., 39:2 (1997), 143-156.

[14] В. В. Жаринов, ТМФ, 140:3 (2004), 355-366.

[15] С.Е. Конштейн, А.Г. Смирнов, И. В. Тютин, ТМФ, 143:2 (2005), 163-194; arXiv: hep-th/0312109.

[16] В. В. Жаринов, ТМФ, 136:2 (2003), 179-196.

[17] Л. Хермандер, Анализ линейных дифференциалъных операторов с частными производными, Т. 1, Мир, М., 1986. 